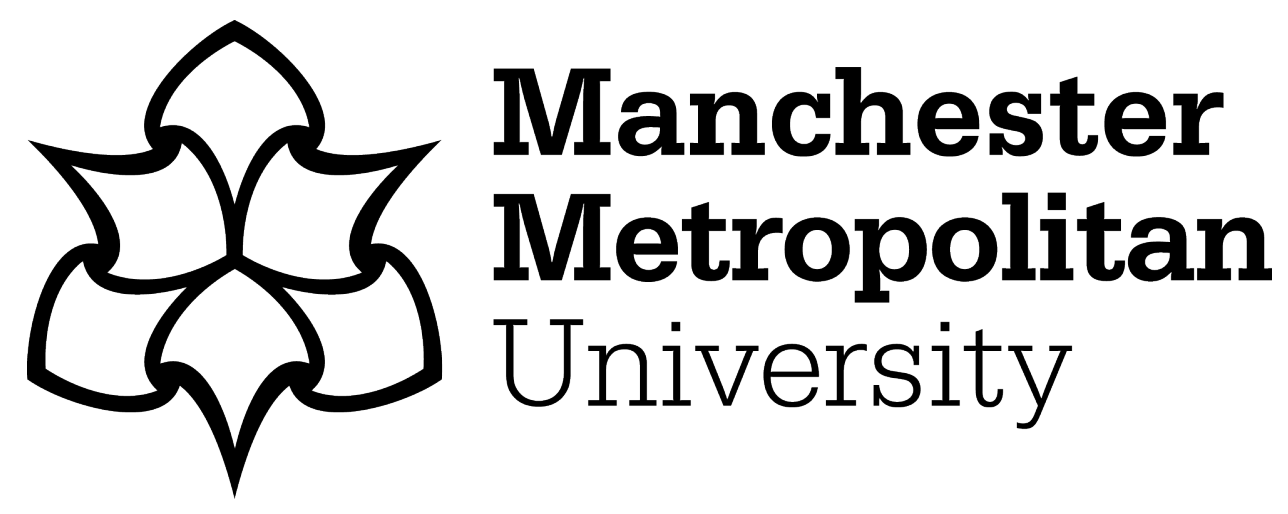

Jiang, SC, Bai, Wei, Cong, PW and Yan, B (2019) Numerical investigation of wave forces on two side-by-side non-identical boxes in close proximity under wave actions. Marine Structures, 63. pp. 16-44. ISSN 0951-8339

Downloaded from: https://e-space.mmu.ac.uk/621442/

Version: Accepted Version

Publisher: Elsevier

DOI: https://doi.org/10.1016/j.marstruc.2018.08.007

Usage rights: Creative Commons: Attribution-Noncommercial-No Derivative Works 4.0

Please cite the published version 


\title{
Numerical investigation of wave forces on two side-by-side non- identical boxes in close proximity under wave actions
}

\author{
Sheng-Chao Jiang, ${ }^{\mathrm{a}, \mathrm{c}, \mathrm{d}, *}$, Wei Bai ${ }^{\mathrm{b}}$, Pei-Wen Cong ${ }^{\mathrm{c}}$, Bin Yan ${ }^{\mathrm{d}}$ \\ a School of Naval Architecture, State Key Laboratory of Structural Analysis for Industrial Equipment, Dalian University of Technology, Dalian, \\ 116024, China \\ b School of Computing, Mathematics and Digital Technology, Manchester Metropolitan University, Chester Street, Manchester, M1 5GD, UK \\ c State Key Laboratory of Coastal and Offshore Engineering, Dalian University of Technology, Dalian, 116024, China \\ ${ }^{\mathrm{d}}$ Department of Civil and Environmental Engineering, National University of Singapore, Singapore, 117576, Singapore
}

\section{A R T I C L E I N F O}

\section{Keywords:}

Fluid resonance

Narrow gap

Wave force

Non-identical

OpenFOAM

\begin{abstract}
A B S T R A C T
Wave forces on two side-by-side non-identical boxes in close proximity under wave actions are investigated by employing a numerical wave flume based on the OpenFOAM ${ }^{\circ}$ package. The similarity and discrepancy of hydrodynamic behavior between the wave response in the narrow gap and the wave forces on the boxes are the focus of the present study. Around resonant frequencies, the large-amplitude piston-type free surface oscillation in the narrow gap can lead to the peak values in the horizontal and vertical wave forces on the downstream box, and the horizontal wave forces on the upstream box. However, only a rapid decrease with the incident wave frequencies can be observed for the vertical wave forces on the upstream box. The resonant frequencies of the wave forces on two boxes are also different with those of wave response in the narrow gap. With the increase of incident wave amplitude, the resonant frequencies and normalized amplitudes of wave forces on downstream box tend to be smaller, which is similar with that of wave response in the narrow gap. However, the normalized wave forces on the upstream box around resonant frequencies do not always decrease with the increase of incident wave amplitude. On the whole, the hydrodynamic behavior of wave forces has some similar characteristics with that of wave response in the narrow gap. However, evident discrepancy between them can also be observed because the other factors, such as the wave response upstream and downstream the two-box systems, also has the non-negligible contribution to wave forces.
\end{abstract}

\section{Introduction}

In the field of offshore and ocean engineering, one of the hydrodynamic issues of crucial importance in the design of Floating Liquid Natural Gas (FLNG) or Floating Production Storage and Off-loading (FPSO) systems is wave induced interactions of a shuttle tanker approaching the FLNG or FPSO. The close proximity of the side-by-side barges is able to generate large-amplitude piston-type free surface oscillations in the narrow gap under wave actions, leading to radical variations of hydrodynamic forces on the barges comparing with that for the same barge in isolation. These extreme waves and resultant hydrodynamic forces would threaten the safety of engineering operations, seriously. An understanding of the mechanisms of hydrodynamics is required in order to achieve the increased safety during loading and off-loading operations.

\footnotetext{
* Corresponding author. School of Naval Architecture, State Key Laboratory of Structural Analysis for Industrial Equipment, Dalian University of Technology, Dalian, 116024, China.

E-mail address: jiangshengchao@foxmail.com (S.-C. Jiang).
} 
Under these circumstances, the topic of gap resonance mentioned above has been receiving increasing attentions nowadays. The large-amplitude piston-like free surface oscillation in the narrow gap in fact shares some similar features with the moonpool resonance problem, for which an analytical solutions was derived by Ref. [15] for the resonant modes and the corresponding modal shapes [4]. proposed a domain decomposition approach for solving the piston-like modal resonance in a two-dimensional moonpool induced by two heaving rectangular hulls [22]. investigated the three-dimensional free surface piston- and sloshing-modal resonant behavior of closely spaced vessels by using the first and second-order wave diffraction analysis in frequency domain, where the lateral and longitudinal modal resonance in waves from any directions were discussed. A fully nonlinear potential flow model was adopted by Ref. [5] for the wave resonance between the side-by-side barges. Numerical simulations suggested that the resonant frequency slightly shifts to higher values as incident wave steepness increases, equivalent to a stiff spring in a nonlinear mass-spring system. Extensive comparisons have demonstrated that the potential flow model is capable of predicting the resonant frequencies and capturing the resonant modes, but the resonant amplitudes have been also reported to be over-predicted compared to the laboratory observations. Many methods were developed for suppressing the unrealistic resonant amplitudes based on the introduction of artificial damping appropriately in the potential flow model, such as in Refs. [3,18]; which can be adopted in the commercial software WAMIT ${ }^{\varpi}$ and HydroStar ${ }^{\oplus}$.

Although the unrealistic values can be suppressed by the modified potential flow models, the actual mechanical essence of the gap resonance in fact still cannot be simulated, correctly. It has been reported by Refs. [13,14] that different artificial damping values have to be adopted for wave amplitudes and wave forces, separately, even if the same structures and incident waves are considered. Physical experimental measurement is an efficient but expensive method for this problem, such as in Refs. [8,19,21] and among others. Another alternative method is the Computational Fluid Dynamics (CFD) simulation based on the Navier-Stokes equations, where the satisfying predictions and the real physical process can be obtained. The influence of viscosity and nonlinearity on the forces and waves generated by a floating twin hulls under heave oscillations was investigated by Ref. [1]. Numerical simulations showed that the nonlinear effect on the wave forces is important for the large amplitude oscillation, while the viscous effect on wave forces is also significant due to flow separation [17]. studied the fluid trapped behavior in the narrow gap affected by water depth. It was suggested that the ratio of water depth to body draft is the key factor that affects the resonant response in shallow depth regime [6]. conducted the experimental and numerical investigation for wave resonance in moonpools with various inlet configurations at low forward speed by heaving excitation. The dependency of piston-mode behavior on forward velocity and heaving amplitude is observed and the flow separation and shed vorticity from the inlets is found to be an important factor. For gap resonance problem, the significant effect of the gap inlet configurations (i.e. sharp and curved corners) on the resonant wave frequency and amplitude can be observed in Ref. [16]. An empirical model was also proposed to predict wave resonance frequency for different curvatures of the gap inlet [23]. considered the resonant fluid response in the narrow gap between two identical fixed rectangular boxes under the excitation of NewWave-type transient wave groups according to the laboratory observations. The higher-order harmonic components were separated and the nonlinear wave-wave and wave-structure interactions were investigated for the transient wave groups action.

The above-mentioned research efforts are mainly for the identical floating objects, whereas the objects usually have different sizes in reality, such as the most typical loading or offloading operations of the side-by-side arrangement between FPSO and LNG vessels [11]. investigated the gap resonance problem caused by the non-identical two-box system, in which the wave amplitude in the narrow gap, the reflection, transformation and energy-loss coefficients are considered. Numerical simulations suggested that the nonidentical nature of the system can not only affect the resonant frequency and amplitude in the narrow gap, but also has the significant effect on the process of energy transformation and energy dissipation compared to the identical box systems. An integral comprehensive understanding on the mechanical essence of the gap resonance can be understood from the perspective of energy transformation and energy dissipation. However, another important aspect, the wave forces on the bodies, are not considered in their work. Since the mechanical essence of the energy transformation and energy dissipation show the close dependence on the configurations of those non-identical two-box system, it is expected that the significant effect of the non-identical nature on the wave forces would be observed, which is also the major motivation of this study.

This paper is based on a direct extension of the numerical method presented by Ref. [11] for wave response in the narrow gap problem. In this study, the wave forces on two-box system with various box drafts are investigated in a viscous numerical wave flume based on the OpenFOAM ${ }^{\circledR}$ package, in which the Naiver-Stokes equations are employed for the governing equations of incompressible two-phase flows. Although the wave forces on two boxes might share some similarities with the hydrodynamic features of wave response in the gap presented in Ref. [11]; some differences in hydrodynamic characteristics between them are expected, and will be highlighted in the present study. The Volume of Fluid (VOF) method [7], documented by Ref. [2]; is adopted to capture the free surface motion, especially the large-amplitude piton-like free surface oscillation in the narrow gap. The relaxation zones by Ref. [9] are equipped to generate the incident wave and eliminate the transmission wave at the inlet and outlet boundaries, respectively, in which the internal wave reflections can also be avoided in this method. More detailed information of the formulations and numerical schemes described above have been given in the previous paper [11]. About the basic numerical implementations of CFD simulations in OpenFOAM ${ }^{\oplus}$, the readers may refer to [10] and [20]. The classical linear potential flow model is also adopted in this study for the purpose of comparison. The main motivation of the work is the numerical analysis of the behaviors of wave forces on the boxes under various influencing factors, especially when the gap resonance happens, by which some dynamic mechanisms of gap resonance can be revealed.

The rest of the present paper is organized as follows: the geometry of numerical simulations adopted in this work is setup and validated against available experimental and numerical data in Section 2 and 3, respectively. The numerical results and discussions are presented in Section 4 to analyse the effect of wave parameters and body configurations on the wave forces of two boxes, especially around resonant frequencies. Numerical investigations include the comparisons of wave forces between the linear potential 


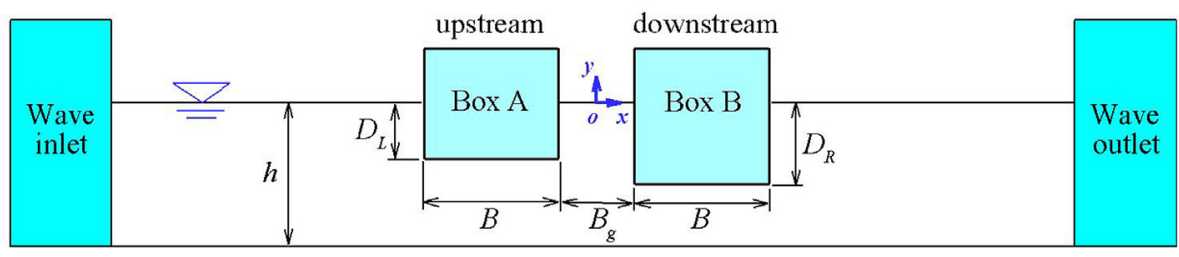

Fig. 1. Sketch of the definition of numerical wave flume.

flow model and the present CFD model, the influence of side-by-side configurations, that is, gap breadth, upstream and downstream box drafts, on the wave forces of the two-box system, and the variation of wave forces with different incident wave amplitudes, especially around resonant frequencies. Finally, the conclusions of this study are summarized in Section 5.

\section{Numerical setup}

The definition sketch for the numerical simulations in this study is illustrated in Fig. 1, in which the origin of the coordinate system is located at the still water level and the wave is propagating in the positive $x$ direction. Two boxes, defined as Box A and Box B, with the identical breadth $B=0.50 \mathrm{~m}$ but non-identical drafts $D_{L}$ and $D_{R}$ for the upstream (left) and downstream (right) boxes, respectively, are fixed in a wave flume with the water depth $h=0.50 \mathrm{~m}$. A narrow gap with the breadth $B_{g}$ is formed by the two boxes, where the extremely large-amplitude piston-type of fluid resonance can be excited as the incident wave frequency is close to the natural frequency of the confined fluid bulk. In accordance with the requirements, a host of simulation cases are designed to conduct the intended investigation by varying the values of gap breadth $B_{g}$, and upstream and downstream box drafts $D_{L}$ and $D_{R}$. The geometry of box drafts $D_{L}$ and $D_{R}$ and the corresponding definition of the test cases are tabulated in Table 1 . Four gap breadths $B_{g}=$ $0.030 \mathrm{~m}, 0.050 \mathrm{~m}, 0.070 \mathrm{~m}, 0.090 \mathrm{~m}$ and three incident wave amplitudes $A_{i}=0.008 \mathrm{~m}, 0.012 \mathrm{~m}$ and $0.016 \mathrm{~m}$ are selected, so totally 192 different cases are considered. The wave frequency $\omega$ is chosen based on the resonant frequency of fluid bulk in the narrow gap. In the following descriptions, the prefix 'Bg' would be adopted for identifying the gap breadth, such as 'Bg50DL103DR252' means $B_{g}=0.050 \mathrm{~m}, D_{L}=0.103 \mathrm{~m}$ and $D_{R}=0.252 \mathrm{~m}$ (see our previous work of [11] for more detailed discussions).

In numerical simulations, the height of the numerical wave flume is fixed at $0.8 \mathrm{~m}$, and the length is closely relevant to the incident wave length $L$ for different simulations. Generally, the length of the relaxation zones are kept at $1.5-2.0 L$; and the distance between relaxation zone to outside of the boxes is $2.0-2.5 \mathrm{~L}$. In addition, $D_{L}$ is not necessarily smaller than $D_{R}$ in this study.

\section{Numerical validation}

The present work is a direct extension of [11] where a large number of validations for wave amplitude in the narrow gap, reflection and transmission coefficients of two-bodies system have already been conducted with the comparison to experimental data in Ref. [21] and numerical results in Ref. [13]. In this section, the numerical wave forces presented in Ref. [14] and the linear potential flow solutions are adopted to further validate the accuracy of present viscous fluid flow model, as the main focus of the present study is on wave forces on two boxes. The mesh dependency tests are carried out firstly by using four different meshes for two cases, Bg30DL103DR103 and Bg70DL252DR252. The detailed mesh information is illustrated in Table 2. To save computational cost, non-uniform meshes are adopted to discretise the computational domain in numerical simulations. The square fine meshes with high resolution are adopted around the boxes, especially in the vicinity of the narrow gap, to accurately capture the large-amplitude piston-like free surface oscillation and to account for the effect of vortex structure in boundary layer. The coarse rectangular meshes with large aspect ratio up to 1/20 (height/length) are equipped in the right relaxation zone for eliminating the transmission wave. As for the left relaxation zone for generating the incident wave, square fine meshes with intermediate resolution are adopted. Typical mesh partitions in the vicinity of the two-box system are depicted in Fig. 2, which is Mesh 1 in Table 2. The drafts $D_{L}$ and $D_{R}$ are denoted as $D$ in this section for simplification since only the cases of $D_{L}=D_{R}$ are examined for validation.

The resonant frequencies of free surface oscillation in the narrow gap can be estimated according to the linear potential flow model. They are 8.00 and $5.10 \mathrm{rad} / \mathrm{s}$ for Bg30DL103DR103 and Bg70DL252DR252, respectively. The mesh dependency tests of wave forces are conducted by using these frequencies, together with the incident wave amplitude $A_{i}=0.012 \mathrm{~m}$. Figs. 3 and 4 depict the typical records of normalized horizontal and vertical wave forces on Boxes A and B, respectively. Clearly steady states of wave force evolutions can be observed in these figures, indicating that the relaxation zones can work well in eliminating the transmission waves

Table 1

List of test cases and corresponding configurations in the present study.

\begin{tabular}{lllll}
\hline & $D_{R}=0.103 \mathrm{~m}$ & $D_{R}=0.153 \mathrm{~m}$ & $D_{R}=0.202 \mathrm{~m}$ & $D_{R}=0.252 \mathrm{~m}$ \\
\hline$D_{L}=0.103 \mathrm{~m}$ & DL103DR103 & DL103DR153 & DL103DR202 & DL103DR252 \\
$D_{L}=0.153 \mathrm{~m}$ & DL153DR103 & DL153DR153 & DL153DR202 & DL153DR252 \\
$D_{L}=0.202 \mathrm{~m}$ & DL202DR103 & DL202DR153 & DL202DR202 & DL202DR252 \\
$D_{L}=0.252 \mathrm{~m}$ & DL252DR103 & DL252DR153 & DL252DR202 & DL252DR252 \\
\hline
\end{tabular}


Table 2

Mesh resolution for convergent test (Elements/Nodes).

\begin{tabular}{|c|c|c|c|c|c|}
\hline$B_{g}(\mathrm{~m})$ & $D_{L}=D_{R}(\mathrm{~m})$ & Mesh 1 & Mesh 2 & Mesh 3 & Mesh 4 \\
\hline 0.030 & 0.103 & $96100 / 194418$ & $130052 / 262726$ & $170400 / 343888$ & $212375 / 428068$ \\
\hline 0.070 & 0.252 & $100896 / 204180$ & $131985 / 266736$ & $162282 / 327676$ & $221737 / 447056$ \\
\hline
\end{tabular}

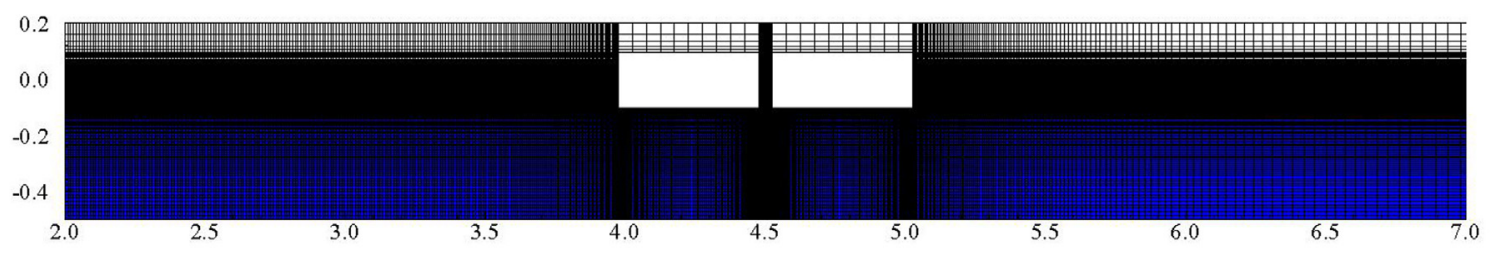

(a) $B_{g}=0.030 \mathrm{~m}, D_{L}=D_{R}=0.103 \mathrm{~m}$

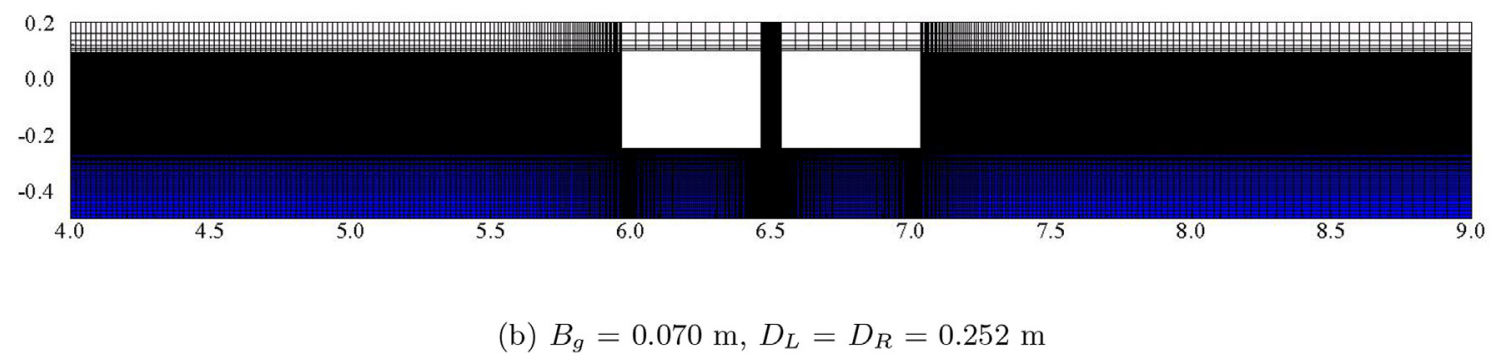

Fig. 2. Typical computational meshes in the vicinity of the two-bodies system.

and avoiding the internal wave reflections. Time signals on different meshes suggest that the discrepancy between them mainly appears at the crest and trough of the wave forces. The convergent solutions can be produced by Mesh 3 for various structures because very little discrepancy between the results of Mesh 3 and Mesh 4 can be observed. Numerical simulations also suggest that the horizontal wave force evolution is quite symmetrical like a sinusoidal function; while a little non-sinusoidal characteristics can be observed for the vertical wave force signal, implying the higher-order harmonic behavior in vertical wave forces. Referring to Bernoulli equation, the vertical wave forces should have close relevant with the square of flow velocity under the boxes bottom, which is the major reason for the nonlinearity.

Furthermore, the normalized amplitudes of wave forces on Boxes A and B around resonant frequencies with four different mesh schemes are depicted in Fig. 5. The amplitudes of wave forces are computed according to the averaged values of force crest and trough between the duration of 40-60 s. The sensitivity analysis by varying the time-windows to the duration of $60-80 \mathrm{~s}$ shows negligible influence on the wave force amplitudes, indicating that the steady state has been reached after $t=40 \mathrm{~s}$. Fig. 5 shows the variation of wave forces with the number of cells around resonant frequencies, in which the percentages between the adjacent mesh schemes are noted for the difference in wave forces. Numerical results suggest that the variation of mesh density has little effect on the wave forces when the number of cells exceeds $1.6 \times 10^{5}$. According to the numerical analysis mentioned above, it can be confirmed that Mesh 3 is able to produce convergent wave forces, and hence it is adopted as the baseline for the following numerical investigations.

Numerical validations of wave forces are conducted against the available Finite Element Method (FEM) numerical results by Ref. [14] and the linear potential flow solutions. The wave force amplitudes $F_{h}$ and $F_{v}$ are shown in Figs. 6 and 7. According to the comparisons, superior agreement between the present model and FEM model can be obtained, confirming that the present numerical wave flume works well in predicting wave forces on the bodies and is capable of producing the accurate results for hydrodynamic analysis. Nevertheless, the linear potential flow model over-predicts the wave forces on these bodies, demonstrating the indispensable of viscous fluid model.

\section{Numerical results and analysis}

The dependence of horizontal and vertical wave forces on incident wave frequency, incident wave amplitude, gap breadths $B_{g}$, upstream draft $D_{L}$ and downstream draft $D_{R}$ is investigated. As mentioned above, in total 64 different geometric cases with three incident wave amplitudes are considered in this work. The amplitudes of horizontal and vertical wave forces are defined as $F_{h}^{A}$ and $F_{v}^{A}$ on Box A and $F_{h}^{B}$ and $F_{v}^{B}$ on Box B, respectively, which are computed according to the averaged values of force crest and trough 


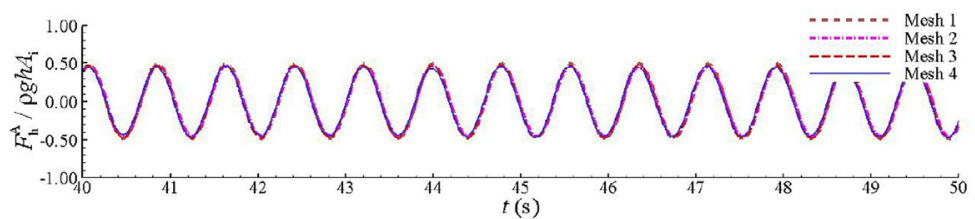

(a) Horizontal force on Box A, $F_{h}^{A}$

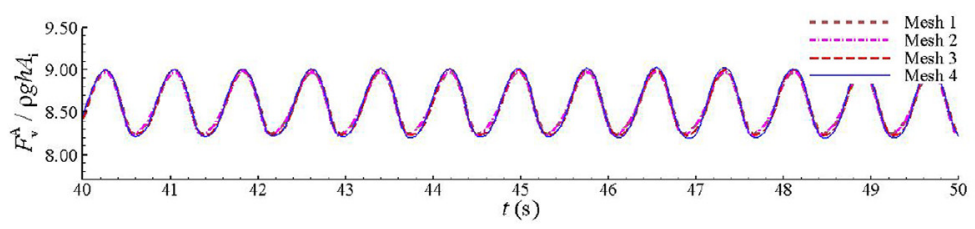

(c) Vertical force on Box A, $F_{v}^{A}$

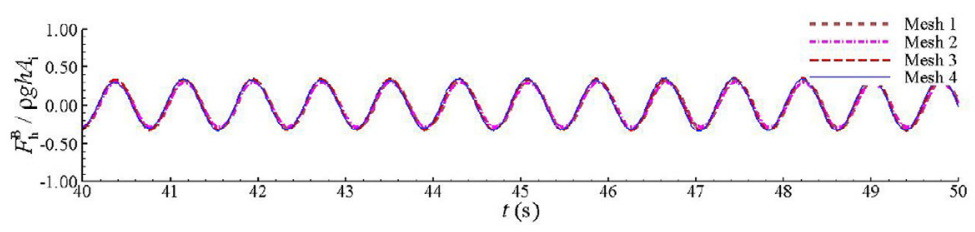

(e) Horizontal force on Box B, $F_{h}^{B}$

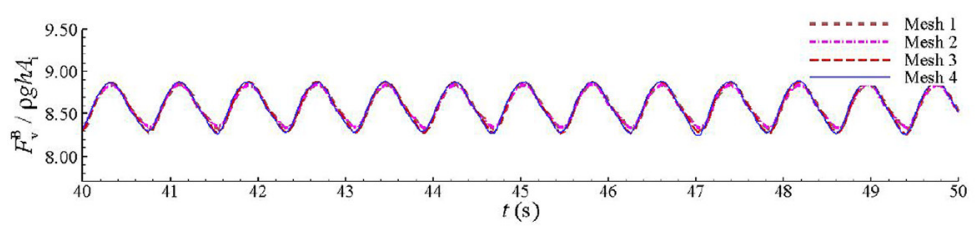

(g) Vertical force on Box B, $F_{v}^{B}$

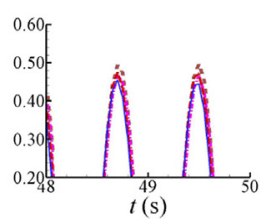

(b)

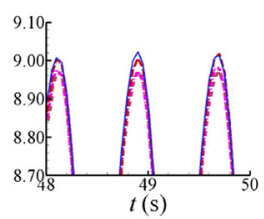

(d)

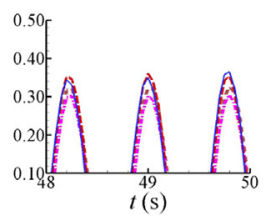

(f)

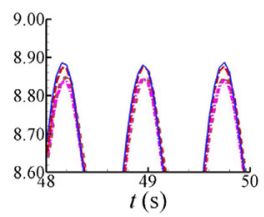

(h)

Fig. 3. Mesh convergent test for gap breadth $B_{g}=0.030 \mathrm{~m}$ and draft $D=0.103 \mathrm{~m}$ with $A_{i}=0.012 \mathrm{~m}$ at resonant frequency $\omega_{g}=8.00 \mathrm{rad} / \mathrm{s}$.

between the duration of 40-60 s. The validation study in the previous section shows that the present numerical wave flume is able to reproduce well the studied scenario of gap resonance between two rectangular boxes. It is employed to investigate the wave forces induced by fluid resonance in the narrow gap formed by two non-identical boxes under wave actions. In order to demonstrate the necessity of employing the viscous fluid model for this problem, the linear potential flow model is also included for the purpose of comparison.

\subsection{General description of wave forces}

Explanations begin with the variation of wave forces on two non-identical boxes with the incident wave frequency. In numerical simulations, the range of incident wave frequencies $\omega$ are from 3.0 to 9.0 with the incident wave amplitude $A_{i}=0.012 \mathrm{~m}$, for the cases of $B_{g}=0.050 \mathrm{~m}$. General comparisons of horizontal and vertical wave forces between present viscous numerical results and linear potential solutions are depicted in Figs. 8 and 9. A three-phase variation of wave forces with incident wave frequencies can be suggested, that is, low frequency range, medium frequency range (around resonant frequency), and high frequency range. The region of medium frequency range is defined as the scope of $\left(\omega_{g} \pm 0.5\right) \mathrm{rad} / \mathrm{s}$, where $\omega_{\mathrm{g}}$ is the resonant frequency of fluid bulk in the narrow gap; while the regions of lower and higher than the medium range can be defined as low frequency range and high frequency range, respectively. Numerical simulations indicate that the potential flow model is able to work well at the scopes of low and high frequency ranges. However, in the medium frequency range, the potential flow model significantly over-predicts the wave forces, especially at resonant frequencies, which is similar to the results of wave response. The assumption of inviscid fluid and irrotational flow in the potential flow model is still the essential reason for the over-prediction. Real physical phenomenon is that the vertex shedding and eddy motion in the narrow gap can reduce the resonant wave amplitude, and consequently lead to the smaller wave forces on boxes, which can be simulated correctly by the viscous fluid model.

Furthermore, numerical analysis is extended to the behavior of wave forces in the medium ranges of frequencies. As shown in 


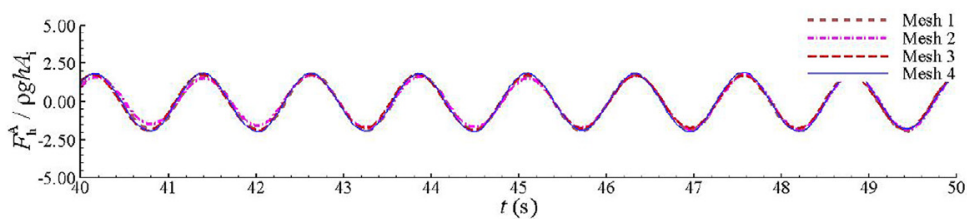

(a) Horizontal force on Box A, $F_{h}^{A}$

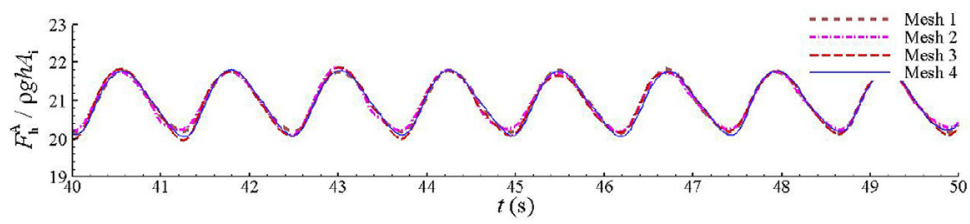

(c) Vertical force on Box A, $F_{v}^{A}$

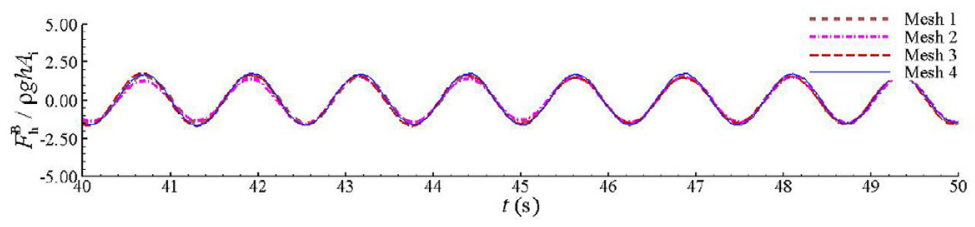

(e) Horizontal force on Box B, $F_{h}^{B}$

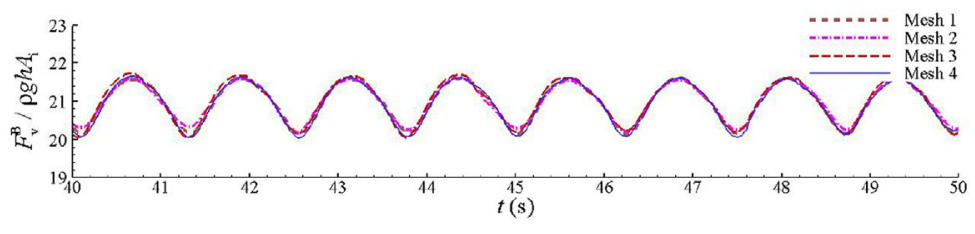

(g) Vertical force on Box B, $F_{v}^{B}$

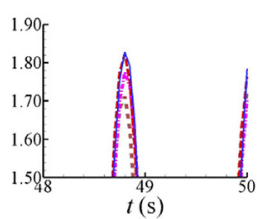

(b)

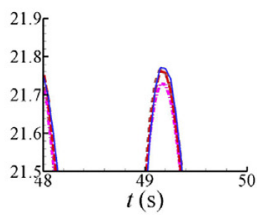

(d)

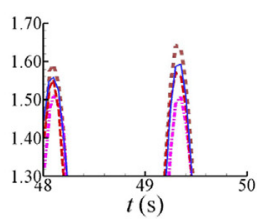

(f)

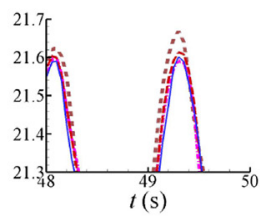

(h)

Fig. 4. Mesh convergent test for gap breadth $B_{g}=0.070 \mathrm{~m}$ and draft $D=0.252 \mathrm{~m}$ with $A_{i}=0.012 \mathrm{~m}$ at resonant frequency $\omega_{g}=5.10 \mathrm{rad} / \mathrm{s}$.

Fig. 8, the maximal horizontal wave forces can be observed around some particular frequencies denoted as the resonant frequencies of waves forces, corresponding to the counterpart of resonant frequencies of fluid oscillation in the narrow gap. However, the resonant frequencies of wave forces are different with that of fluid oscillation [11]. Viscous numerical simulations suggest that the resonant frequencies of $F_{h}^{A}$ are always larger than those of fluid oscillation, while the resonant frequencies of $F_{h}^{B}$ are always smaller than those of fluid oscillation. As an example, the resonant frequency of fluid oscillation in the narrow gap is $\omega=6.6 \mathrm{rad} / \mathrm{s}$ for Bg50DL103DR252, whereas the resonant frequencies of $F_{h}^{A}$ and $F_{h}^{B}$ are 6.9 and $6.4 \mathrm{rad} / \mathrm{s}$, respectively. Many factors can affect the phenomena mentioned above, including the wave response in the narrow gap, wave elevation outside the two-body system, phase difference between them, and vortical flow motion in the vicinity of the narrow gap, etc. In difference to the viscous fluid flow analysis, the exaggerated wave amplitudes in the narrow gap at resonant frequencies become the controlling factor of wave forces in potential flow results, and consequently lead to that the maximal wave forces always occur at resonant frequencies of the fluid oscillation. It is not the real physical hydrodynamic behavior due to the ignorance of fluid viscosity and flow rotation under the potential flow assumption.

Fig. 9 shows the vertical wave forces on two boxes, $F_{v}^{A}$ and $F_{v}^{B}$, against the incident wave frequencies. A lowest $F_{v}^{A}$ can be observed at the frequency band higher than resonant frequencies in the potential flow solution. It is a fictional response frequency produced by the potential flow model, where the corresponding $F_{v}^{A}$ is underestimated. Viscous fluid flow model manifests a generally decreasing tendency of the vertical forces $F_{v}^{A}$ on Box A with incident wave frequencies at the medium frequencies range. The noticeable peak value in potential flow solutions even disappears in viscous fluid flow model results. As for the CFD wave forces on Box B, the magnitudes of $F_{v}^{B}$ increase firstly, and then approach to the maximal values in the region of the medium frequencies. Further comparisons suggested that the resonant frequencies of $F_{v}^{B}$ are smaller than those of fluid oscillation in the narrow gap in Ref. [11]. In the present numerical simulations, the vertical wave forces on Box A are overall larger than those observed on Box B. The comparisons between Fig. $8 \mathrm{~d}$ and $\mathrm{m}$, and Fig. $9 \mathrm{~d}$ and $\mathrm{m}$ indicate that the total forces on the two boxes are smaller when the larger draft is located in the upstream, which could be a helpful recommendation for the side-by-side operations. 

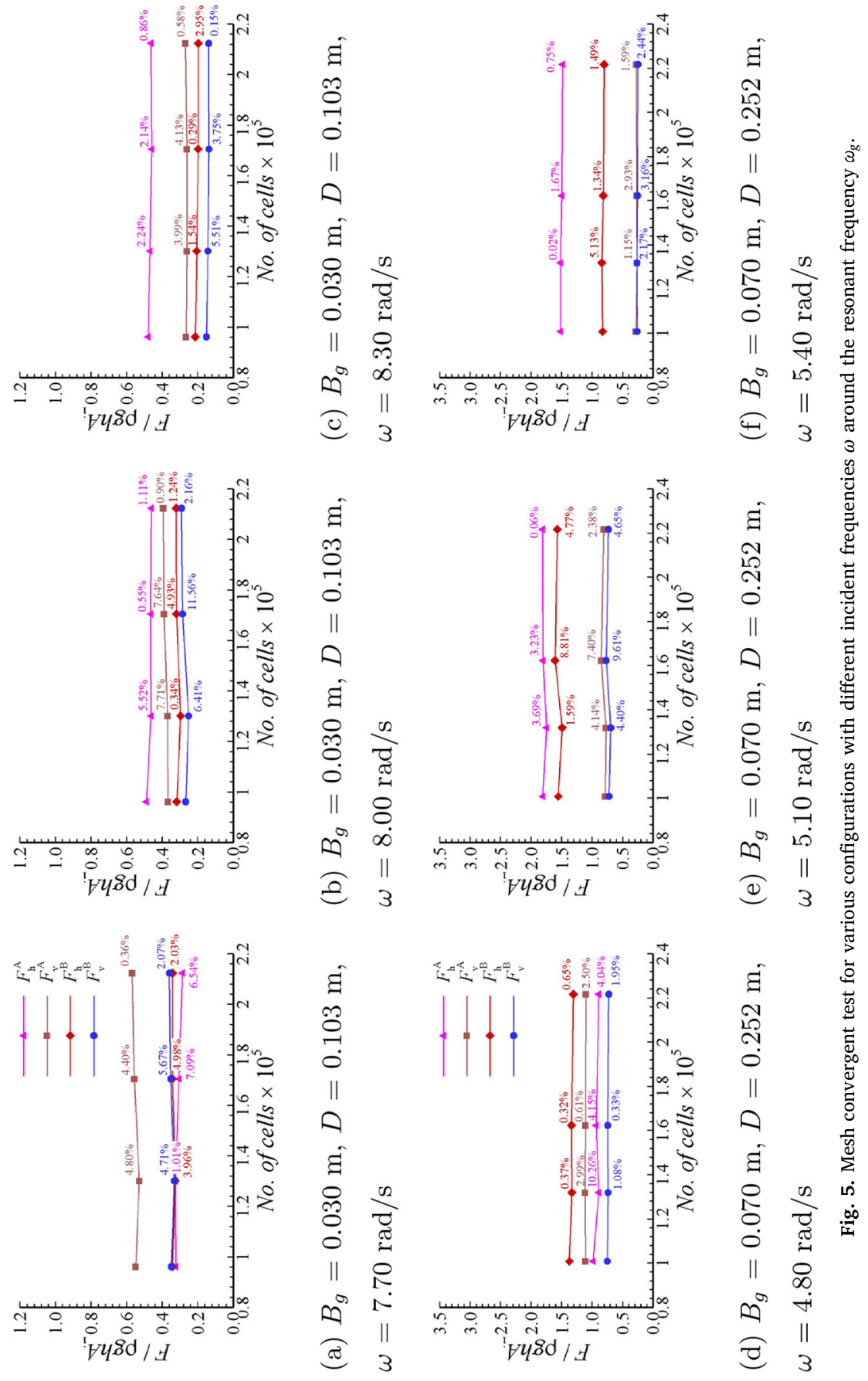

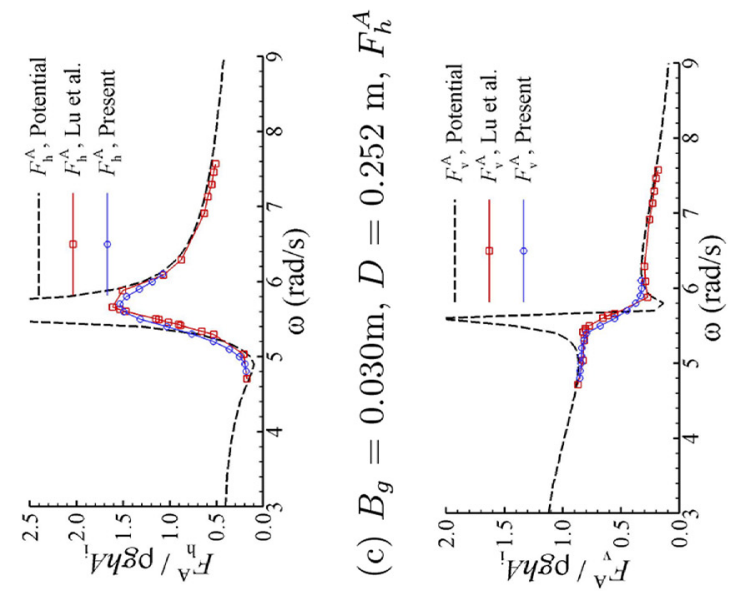

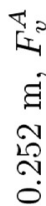

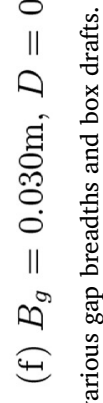

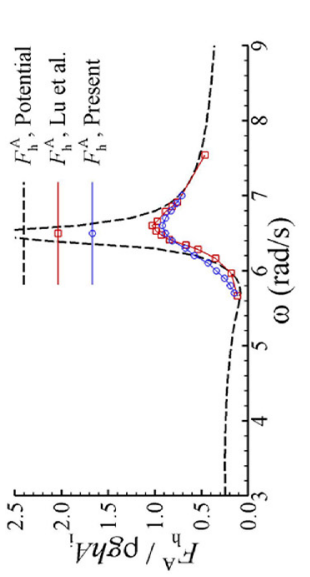

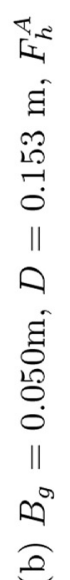

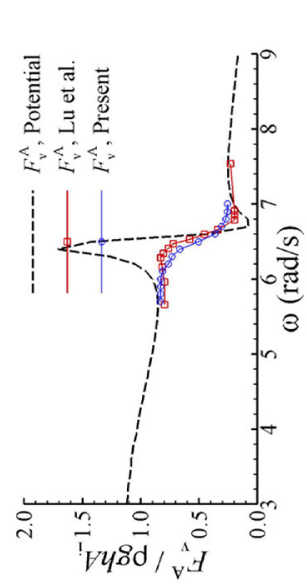

造

हो ठ

마

11

0 .

ह્1

잉

11 氙

(2) ह

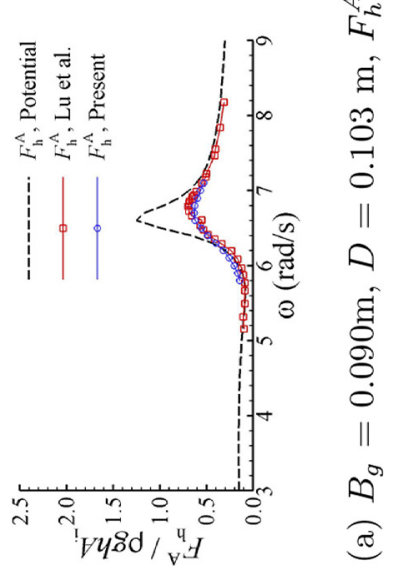

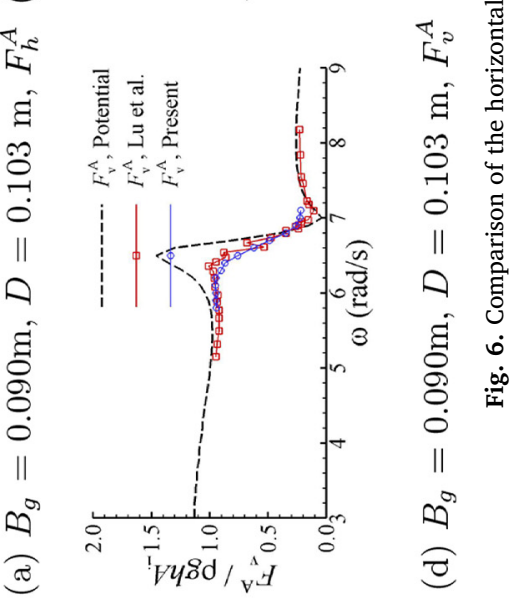




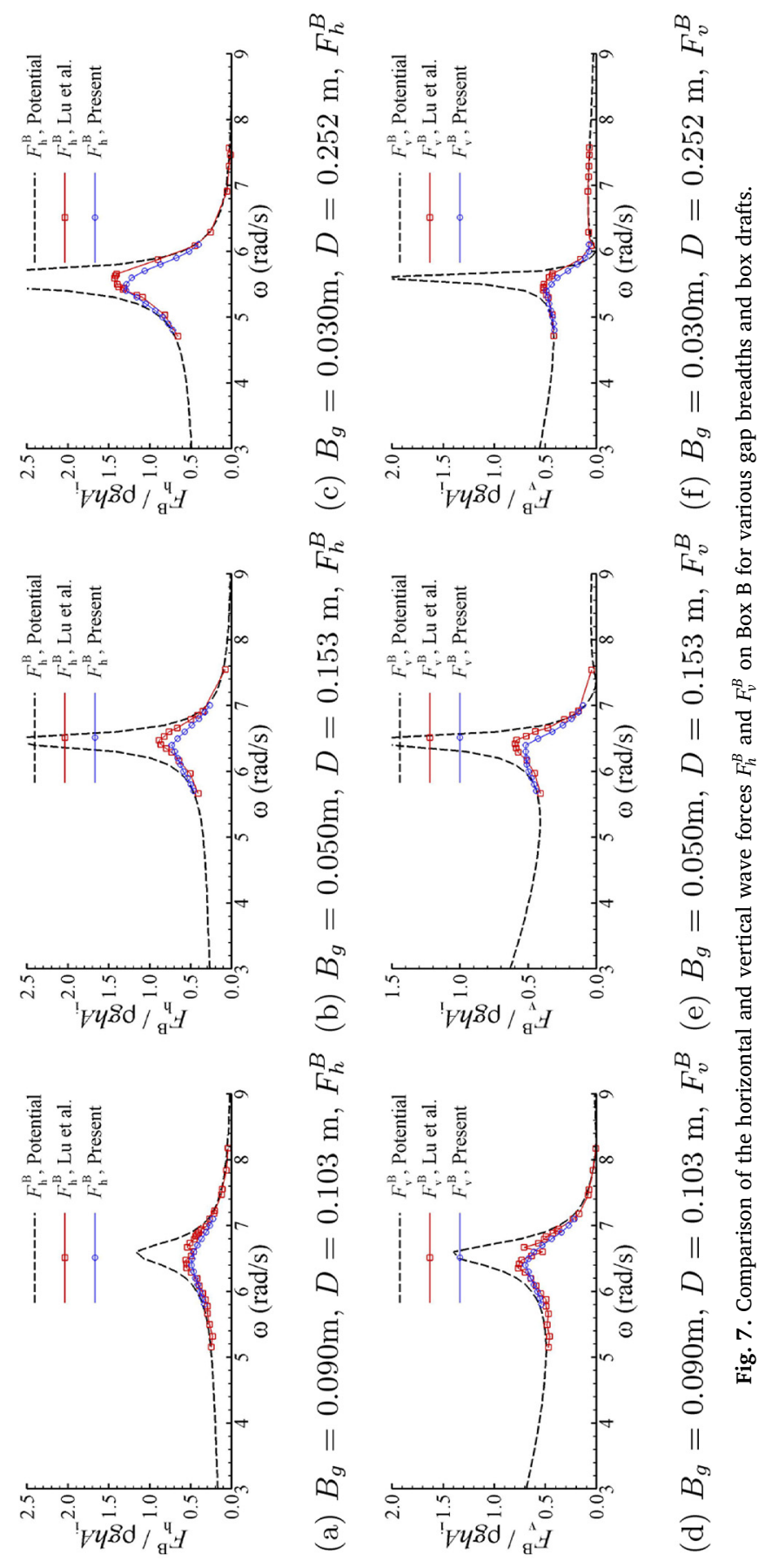



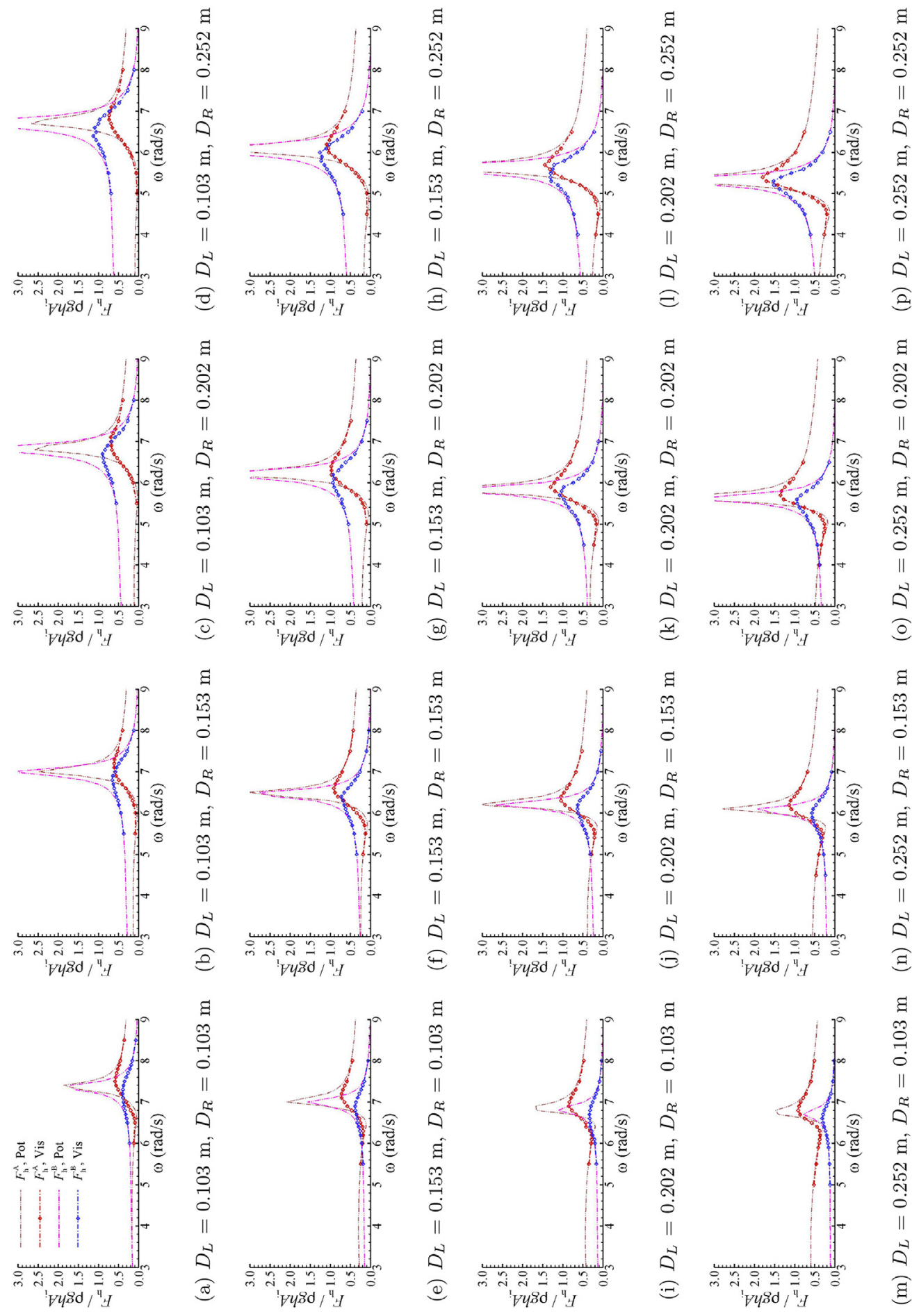

Fig. 8. Comparison of normalized horizontal wave forces $F_{h}^{A}$ and $F_{h}^{B}$ on Boxes A and B under wave actions.

\subsection{Influence of downstream box draft}

In this section, the variation of wave forces with incident wave frequencies, concerning with the influence of downstream box draft, is investigated. All of the cases in Table 1 with the incident wave amplitude $A_{i}=0.012 \mathrm{~m}$ are simulated and the corresponding numerical results of horizontal and vertical wave forces on Boxes A and B are arranged in Figs. 10-13. Besides the CFD solutions, the potential flow solutions are also included for analysing the tendency of wave forces at the scopes of low and high frequencies. A 

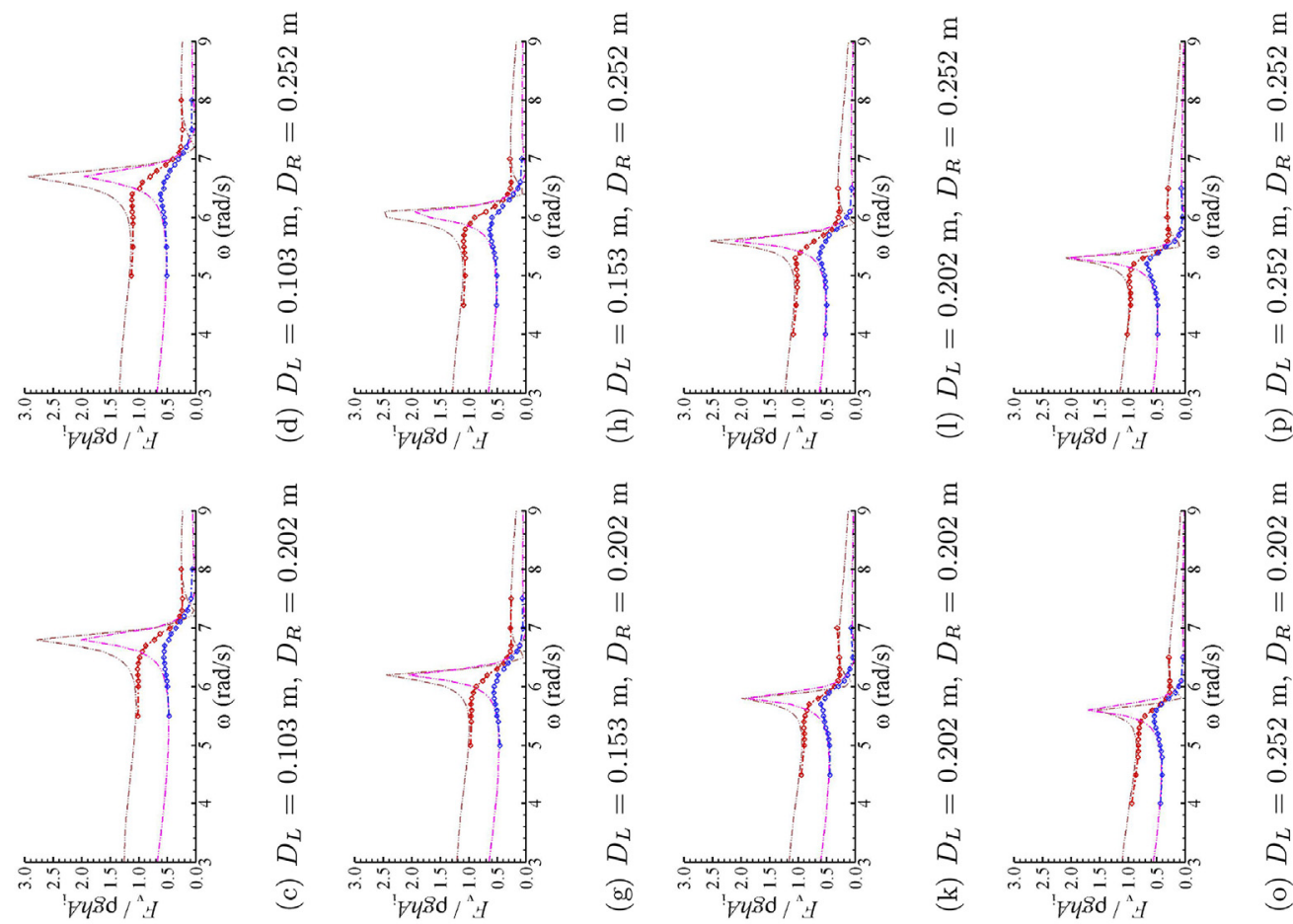

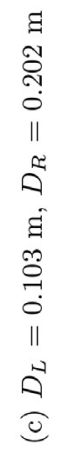

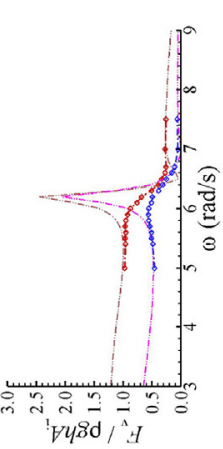

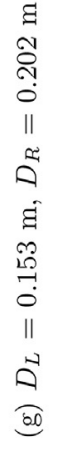
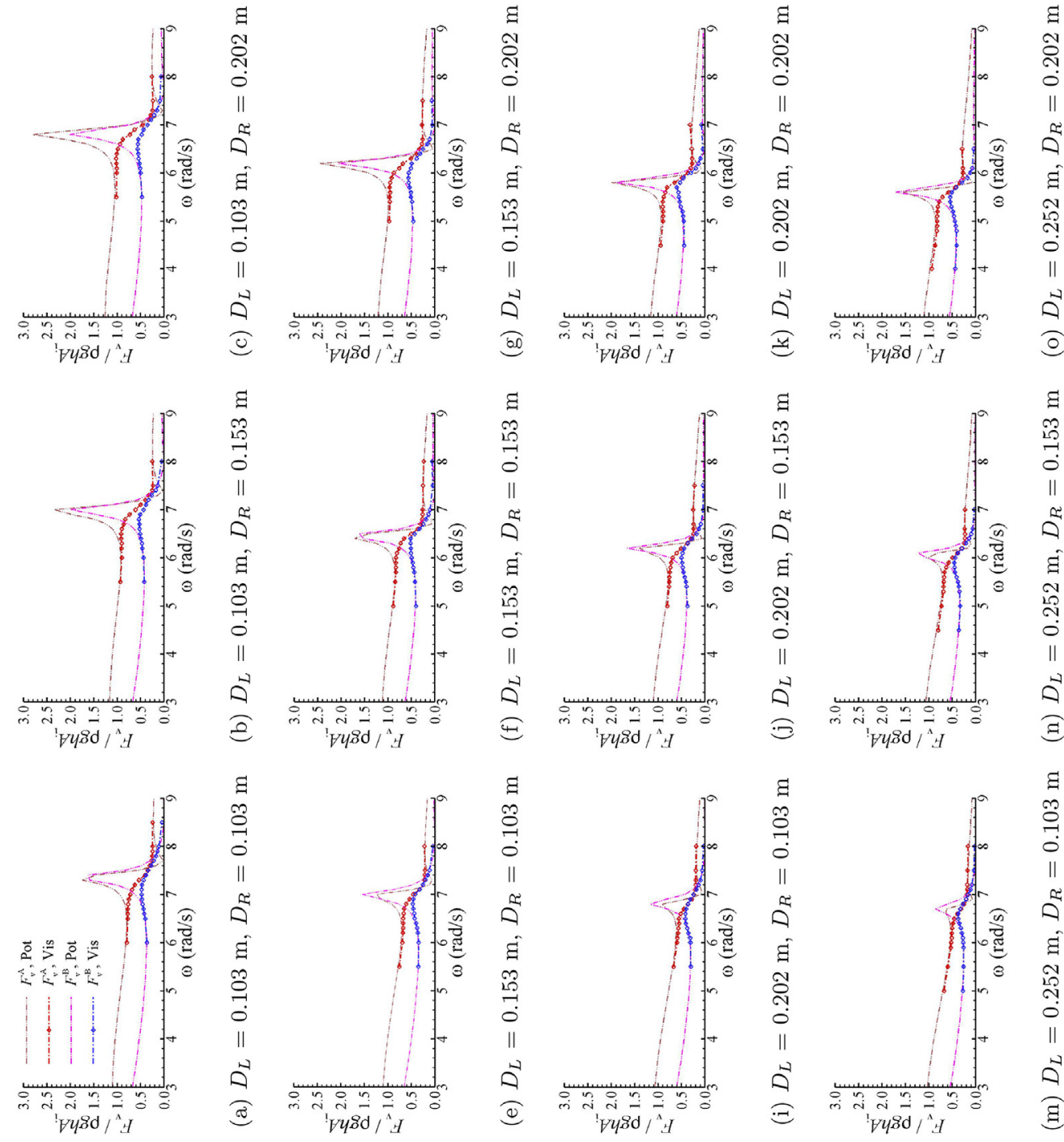

Fig. 9. Comparison of normalized vertical wave forces $F_{v}^{A}$ and $F_{v}^{B}$ on Boxes A and B under wave actions.

general comparison indicates that the wave forces with different downstream box drafts can still be described by the three-phase variation with incident wave frequencies. Numerical analysis is focused on the hydrodynamic behavior of wave forces in the region of medium frequencies, where the essential phenomena of gap resonance happen.

The magnitudes of horizontal wave forces on Boxes $\mathrm{A}$ and $\mathrm{B}$, that is, $F_{h}^{A}$ and $F_{h}^{B}$, for different downstream box drafts, with incident wave frequencies $\omega$ are depicted in Figs. 10 and 11. Numerical simulations illustrate that the resonant frequencies and amplitudes of wave forces on Boxes A and B tend to decrease and increase, respectively, with the increase of downstream box draft. More sensitive 


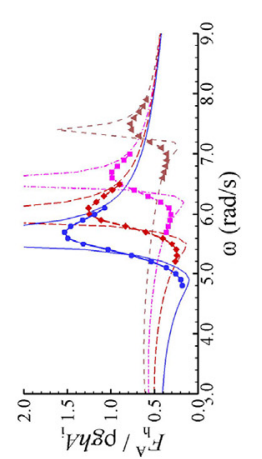

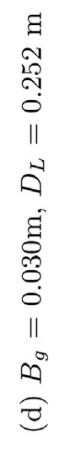
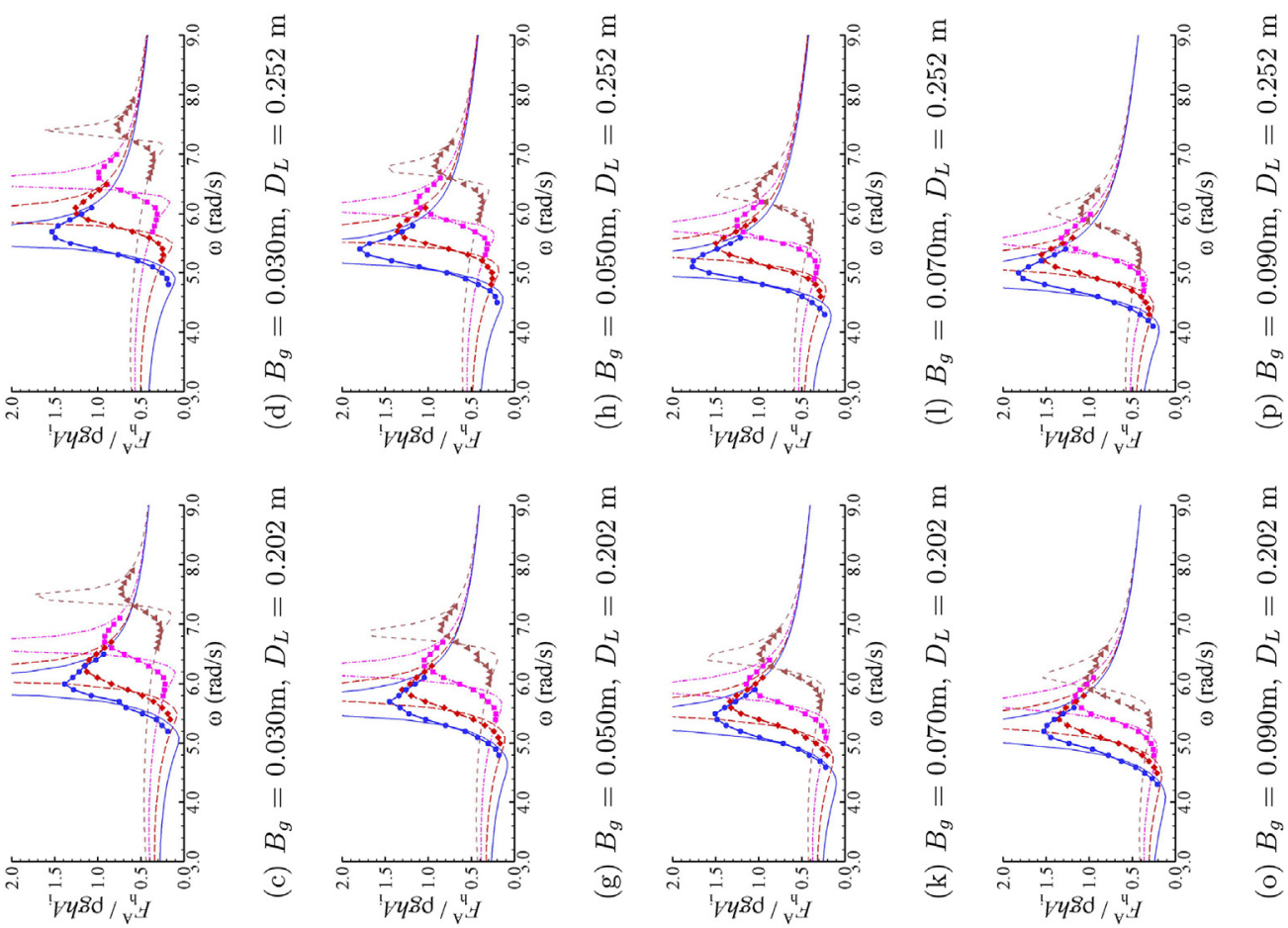

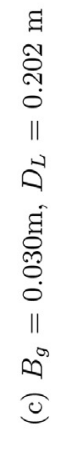
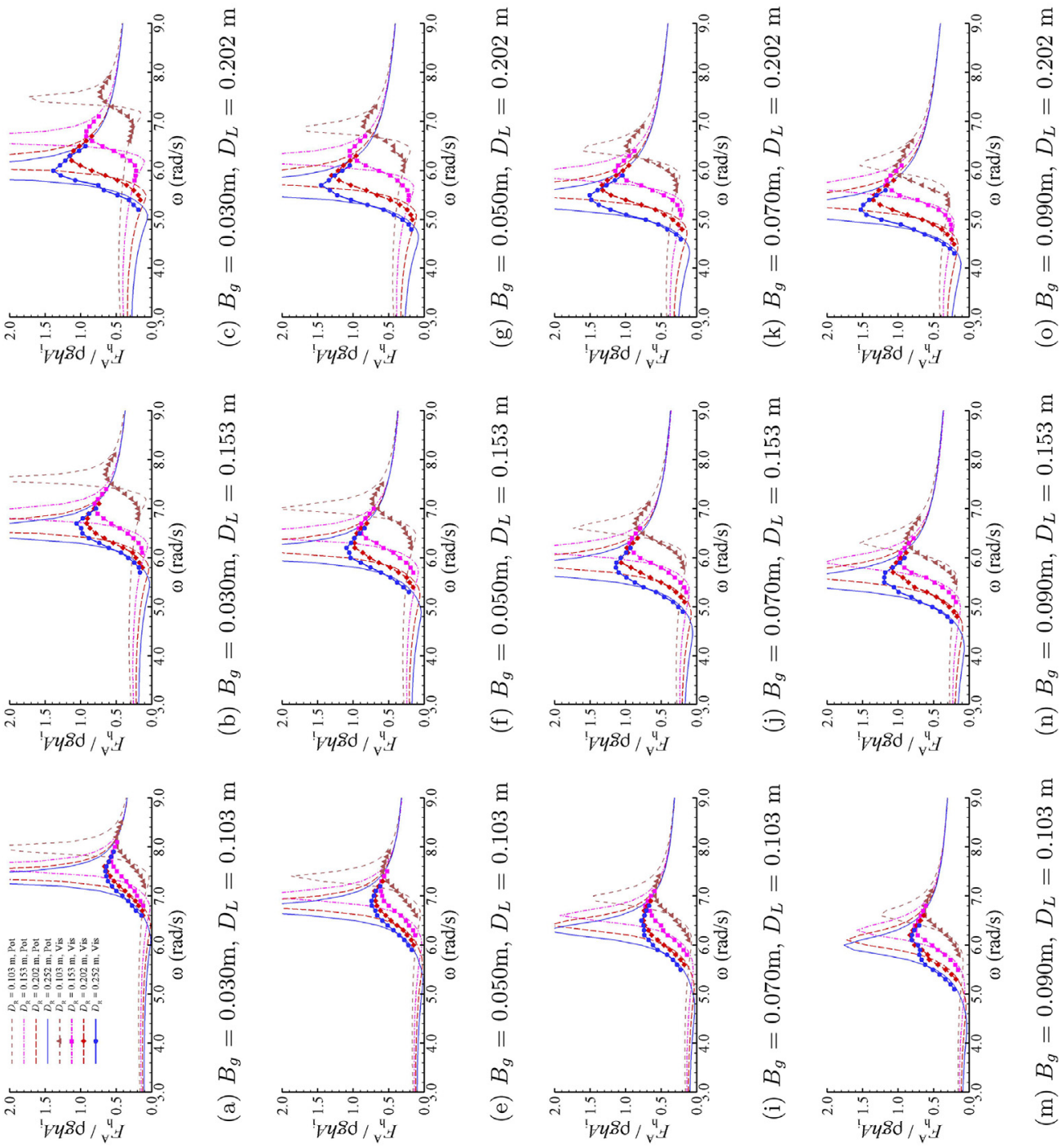

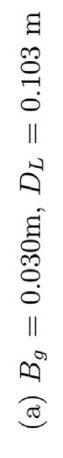
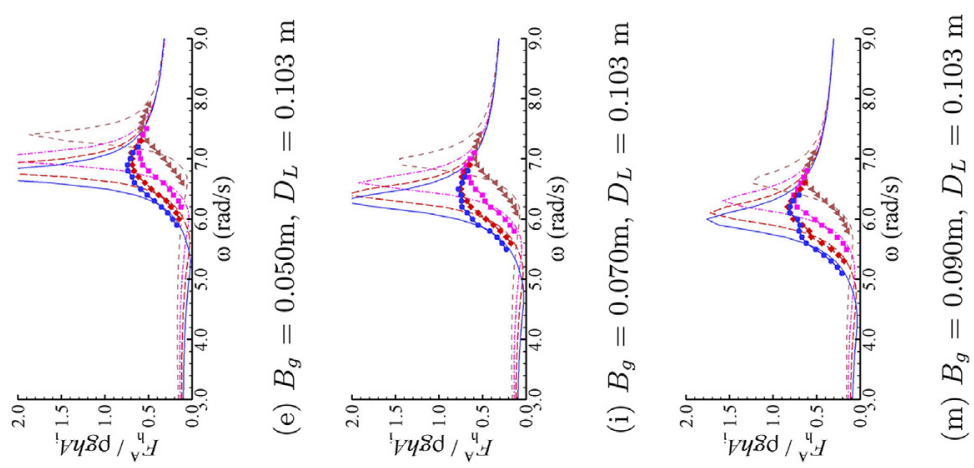

Fig. 10. Comparison of normalized horizontal wave forces $F_{h}^{A}$ on Box A for various downstream box drafts $D_{R}$ under wave actions.

results of wave forces can be observed for large upstream box drafts. It is similar with the results of free surface oscillation by Ref. [11] at medium frequencies, because the wave forces are closely relevant to wave responses in the narrow gap. While the free surface oscillation in the narrow gap is dependent on the vertical velocity at the gap bottom [12]. At the range of low frequencies, the horizontal wave forces on Boxes A and B decrease and increase with the increase of downstream box draft, respectively. As for the region of high frequencies, the insignificant effect of downstream box draft on $F_{h}^{A}$ and $F_{h}^{B}$ can be observed, where $F_{h}^{A}$ gives rise to a constant and $F_{h}^{B}$ approaches to zero with the increase of $\omega$ for different $D_{R}$. 

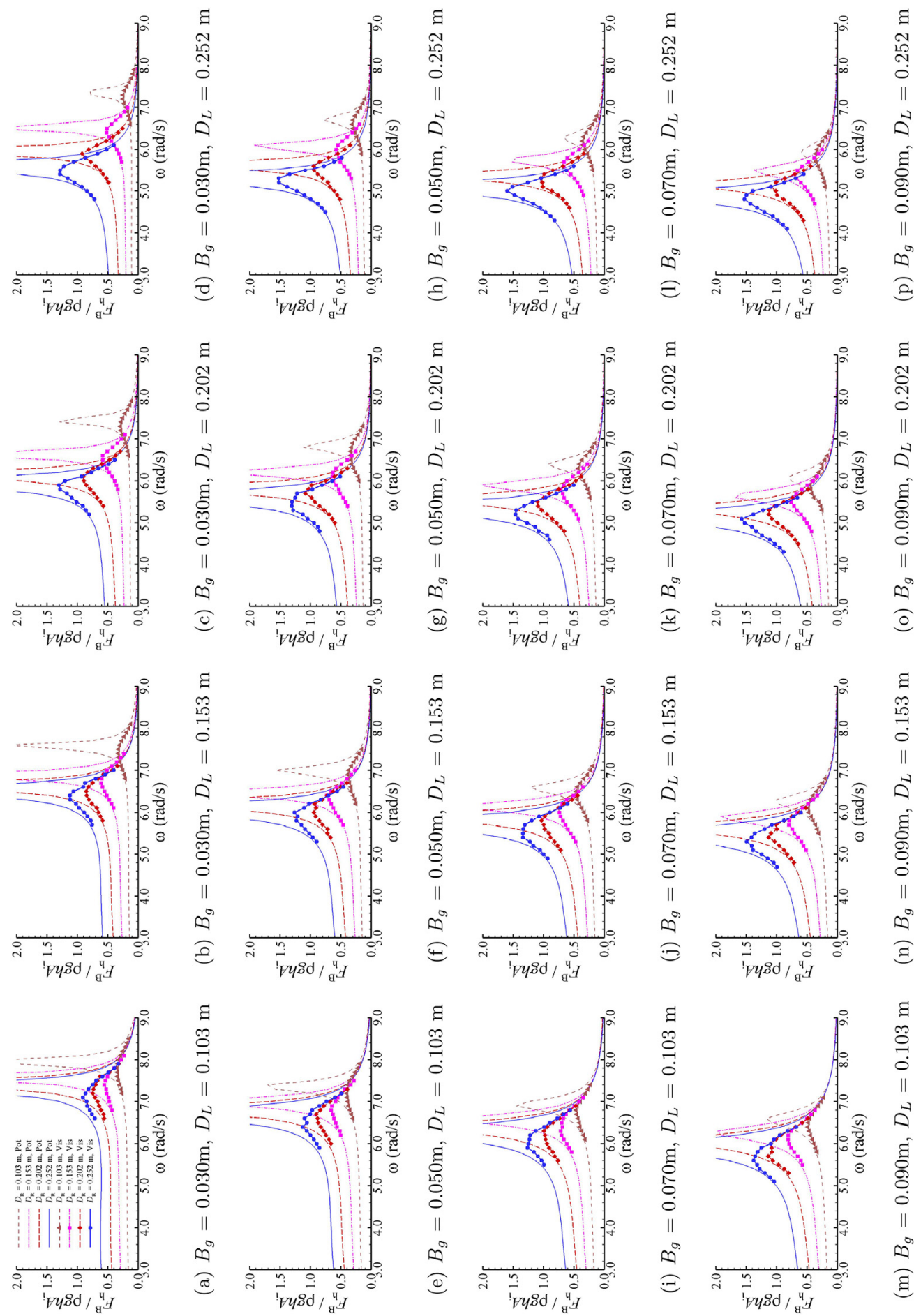

Fig. 11. Comparison of normalized horizontal wave forces $F_{h}^{B}$ on Box B for various downstream box drafts $D_{R}$ under wave actions.

Figs. 12 and 13 investigate the influence of downstream box draft on the vertical wave forces on Boxes A and B, that is, $F_{v}^{A}$ and $F_{v}^{B}$. At the scope of low frequencies, the increase of downstream box draft can lead to the increase of $F_{v}^{A}$ and $F_{v}^{B}$. The insignificant effect of downstream box draft can be obtained at the region of high frequencies, where both $F_{v}^{A}$ and $F_{v}^{B}$ approach to the same for different downstream box drafts. At medium range of incident frequencies, the resonant frequencies and amplitudes of $F_{v}^{B}$ decrease and increase with the increase of downstream box draft, respectively, which is similar with the characteristics of horizontal wave forces on Boxes A and B. Difference to $F_{v}^{B}$, the vertical wave forces $F_{v}^{A}$ on Box A are observed to decrease in general with the increase of 

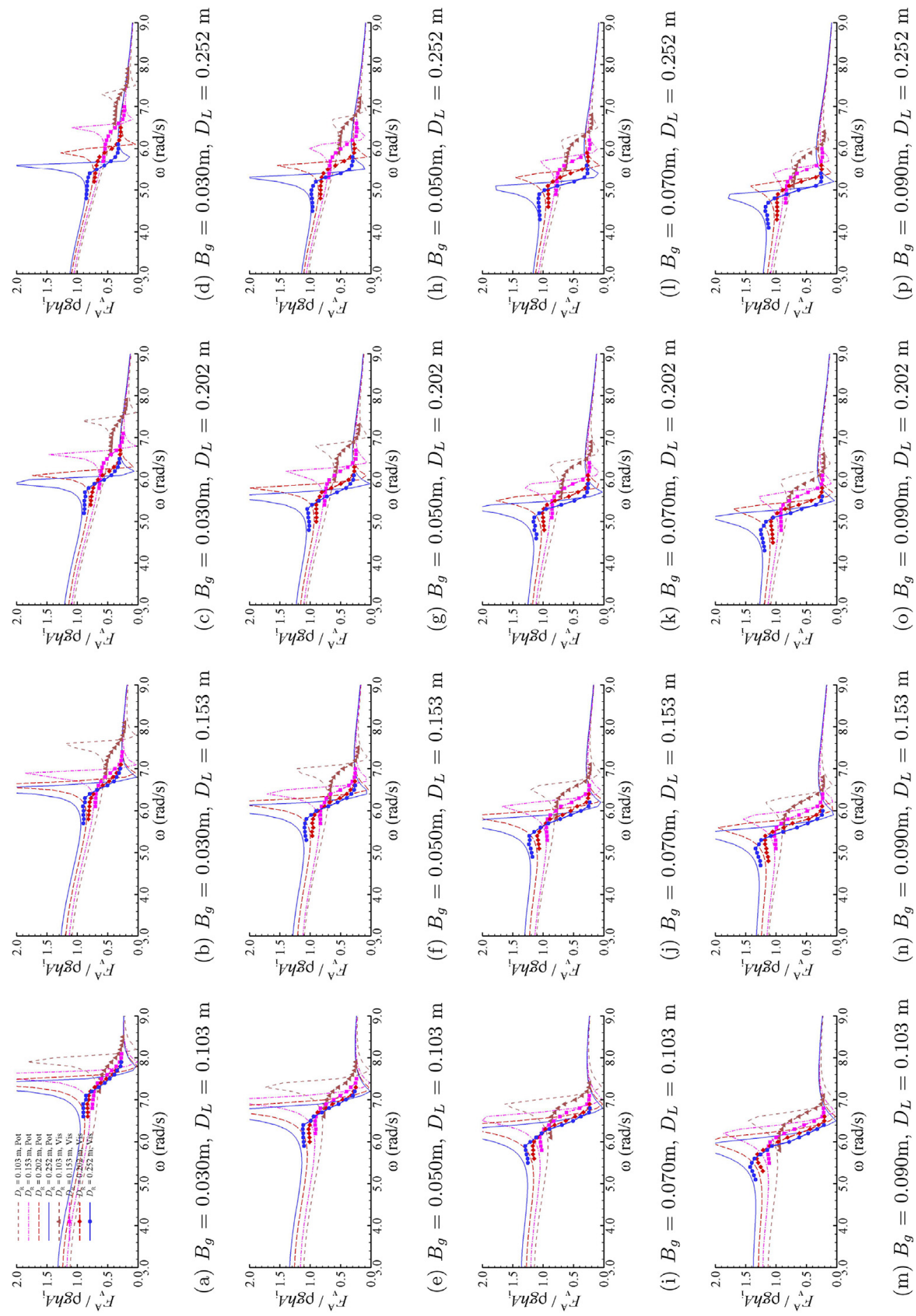

Fig. 12. Comparison of normalized vertical wave forces $F_{v}^{A}$ on Box A for various downstream box drafts $D_{R}$ under wave actions.

incident wave frequencies and drops rapidly at medium frequencies. However, the characteristic frequencies share the similar decreasing tendency with the increase of downstream draft as the vertical wave forces $F_{v}^{B}$ on Box B. It can be seen from the figures in this section that, the variation of wave forces with downstream box draft is more sensitivity when the upstream box draft is large. 

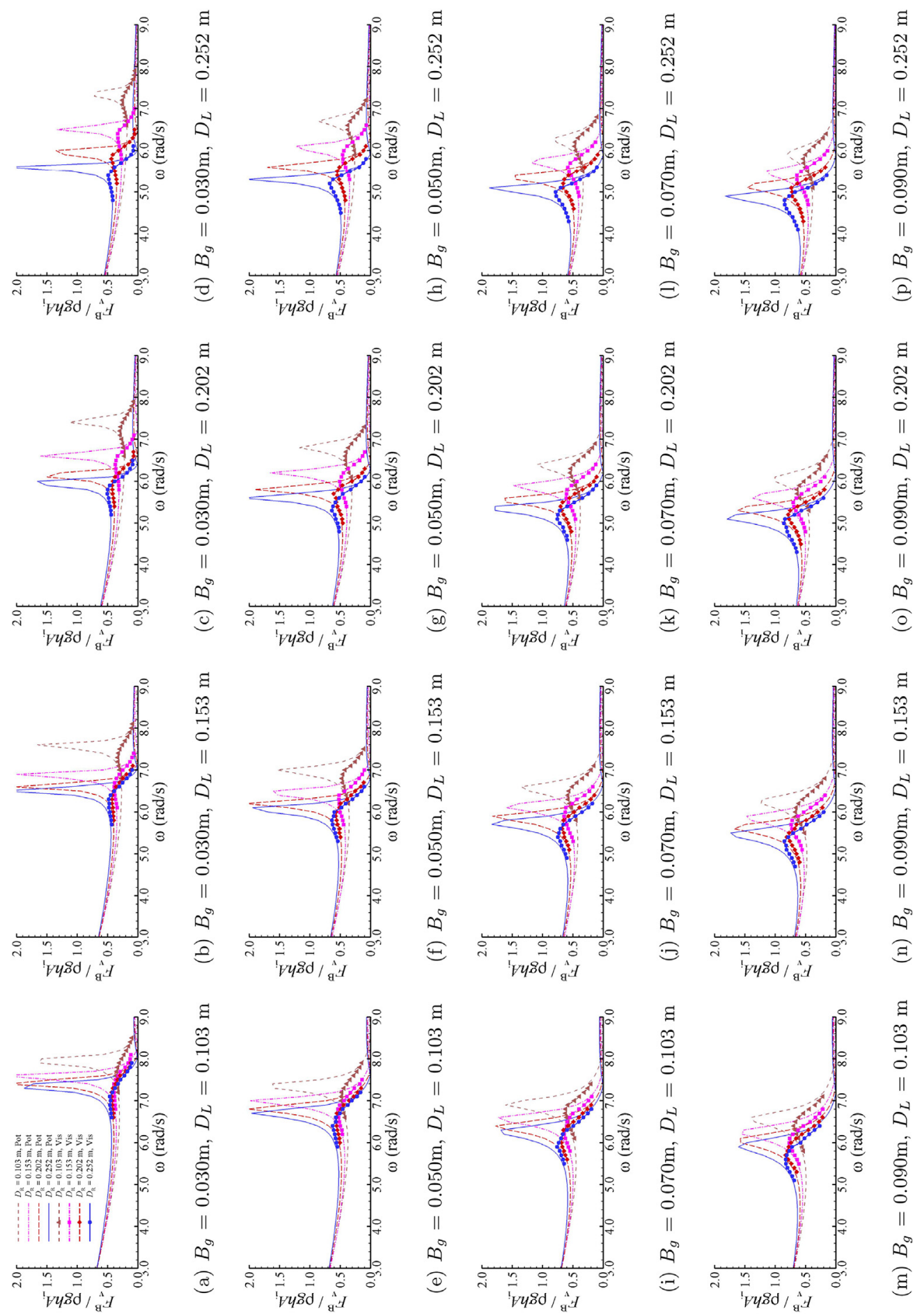

Fig. 13. Comparison of normalized vertical wave forces $F_{v}^{B}$ on Box B for various downstream box drafts $D_{R}$ under wave actions.

\subsection{Influence of upstream box draft}

The influence of upstream box draft on the horizontal and vertical wave forces on Boxes A and B is demonstrated in Figs. 14-17, respectively. It can be seen from these figures that the increase of upstream box draft is able to produce the decreasing resonant frequencies of $F_{h}^{A}, F_{h}^{B}$ and $F_{v}^{B}$. The vertical wave force $F_{v}^{A}$ on Box A has a rapid decline at medium frequencies, and the characteristic frequency also decreases with the increase of upstream box draft. Those variations of the resonant and characteristic frequencies of 


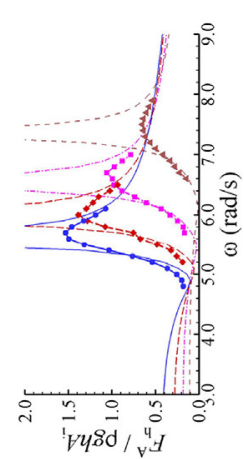

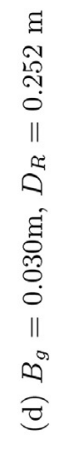
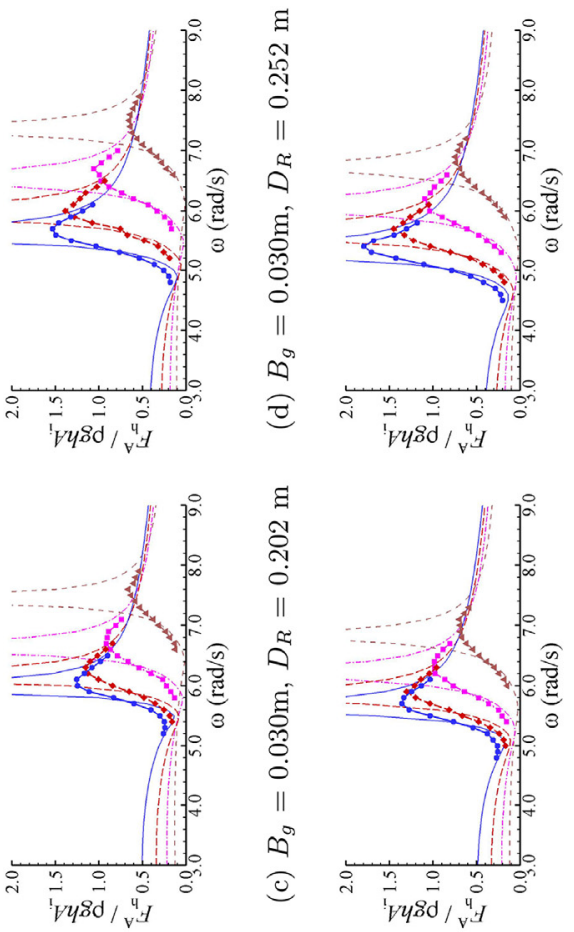

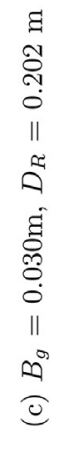
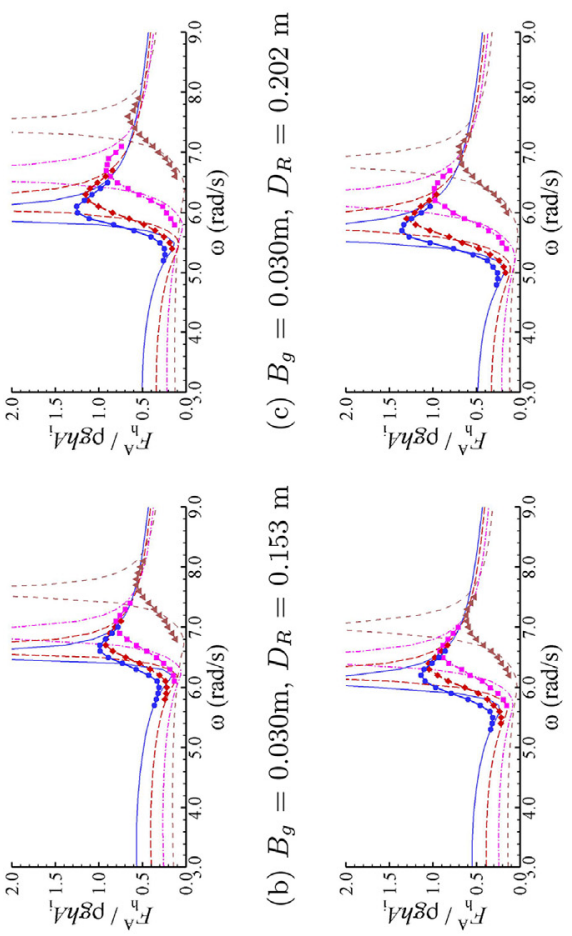

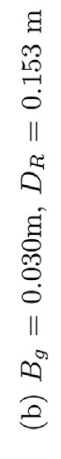
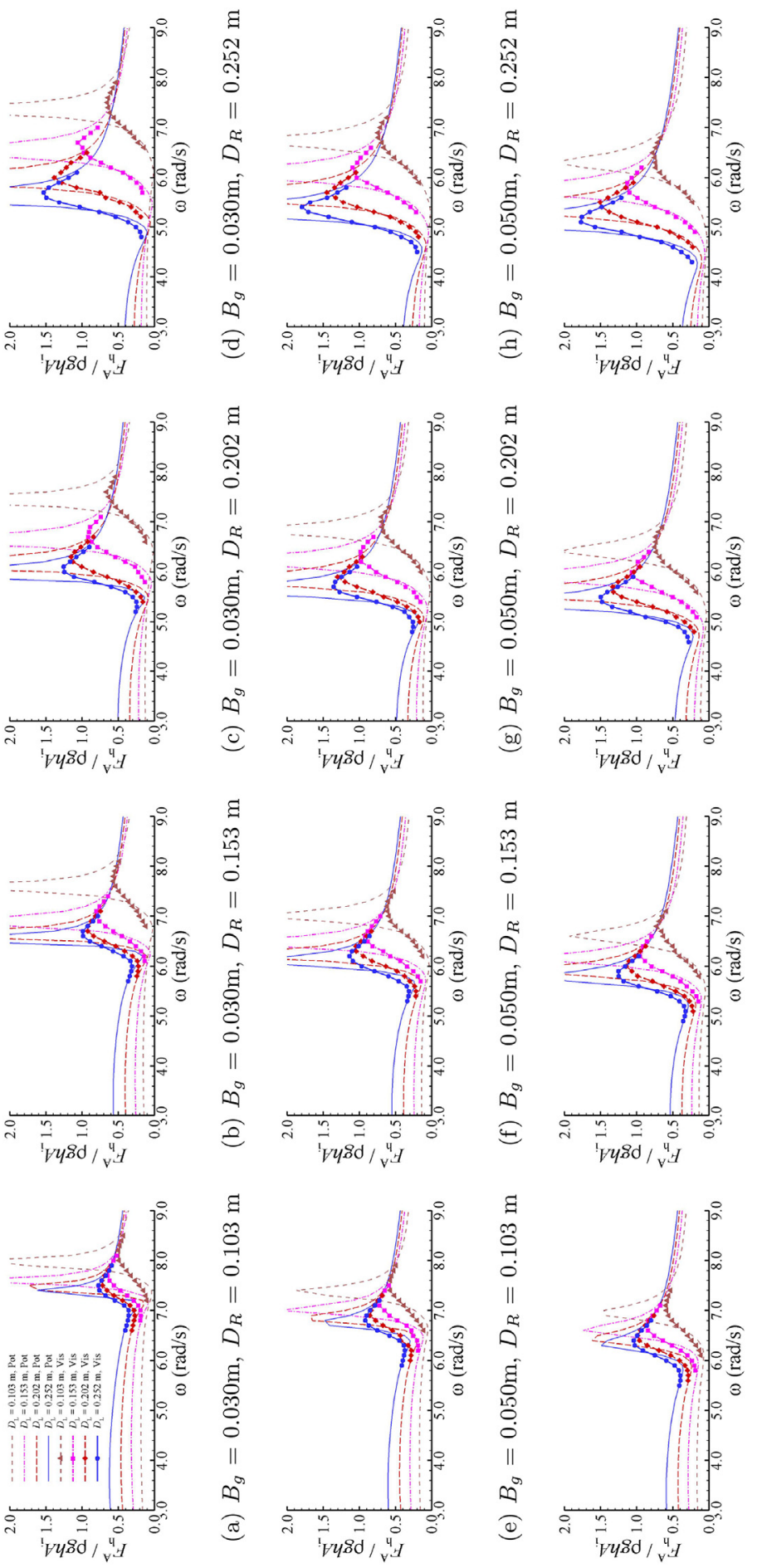
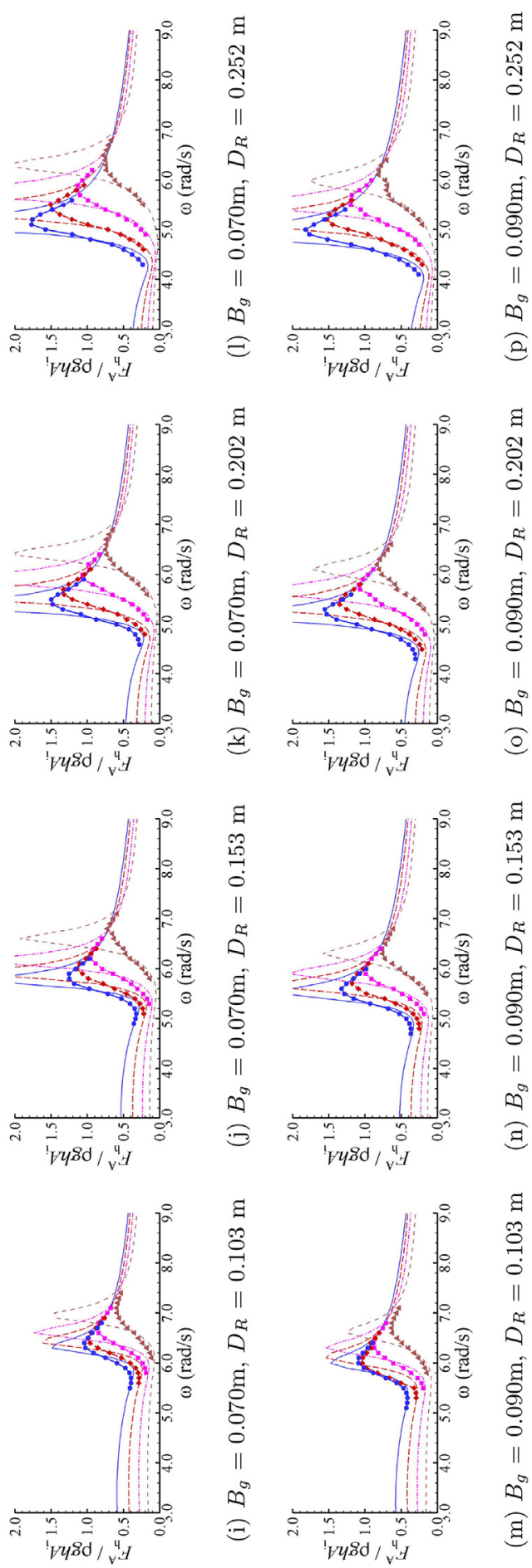

Fig. 14. Comparison of normalized horizontal wave forces $F_{h}^{A}$ on Box A for various upstream box drafts $D_{L}$ under wave actions.

wave forces are similar with the resonant frequencies of wave response in the narrow gap [11]. The close relation between the wave forces and wave responses is the reason for the analogous.

The variations of horizontal and vertical wave forces $F_{h}^{A}$ and $F_{v}^{A}$ on Box A with different upstream box drafts are illustrated in Figs. 14 and 16. Fig. 14 suggests that the horizontal wave forces $F_{h}^{A}$ on Box A increase with the increase of upstream box draft at the regions of low and medium frequencies. With the further increase of incident wave frequency, $F_{h}^{A}$ tends to a constant for various upstream box drafts at the region of high frequencies. The wave response in the upstream of Box A is the main reason for the above 

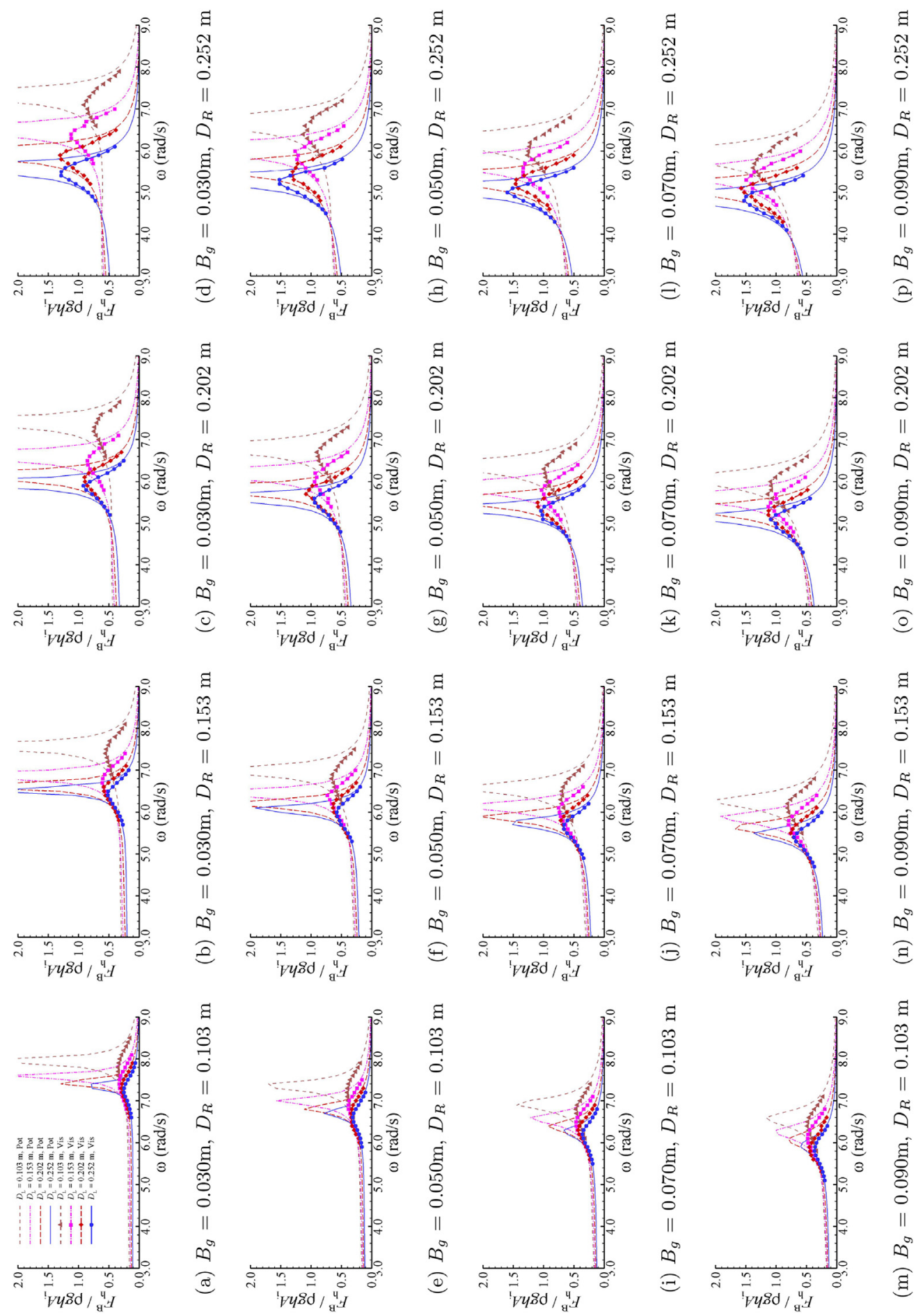

Fig. 15. Comparison of normalized horizontal wave forces $F_{h}^{B}$ on Box B for various upstream box drafts $D_{L}$ under wave actions.

phenomena. The vertical wave forces on Box A in Fig. 16 indicate that the larger upstream box draft can lead to a smaller $F_{v}^{A}$ at low and medium frequencies ranges. The distribution of wave particle velocity, which decreases followed the hyperbolic cosine function along the water depth, is the reason for this phenomenon. At the range of high incident frequencies, $F_{v}^{A}$ approaches to a constant for different upstream box drafts.

In order to examine the influence of upstream box drafts on the horizontal and vertical wave forces on Box B, the variations of $F_{h}^{B}$ and $F_{v}^{B}$ with upstream box drafts are shown in Figs. 15 and 17. Three kinds of situations about the effect of upstream box drafts $D_{L}$ can 

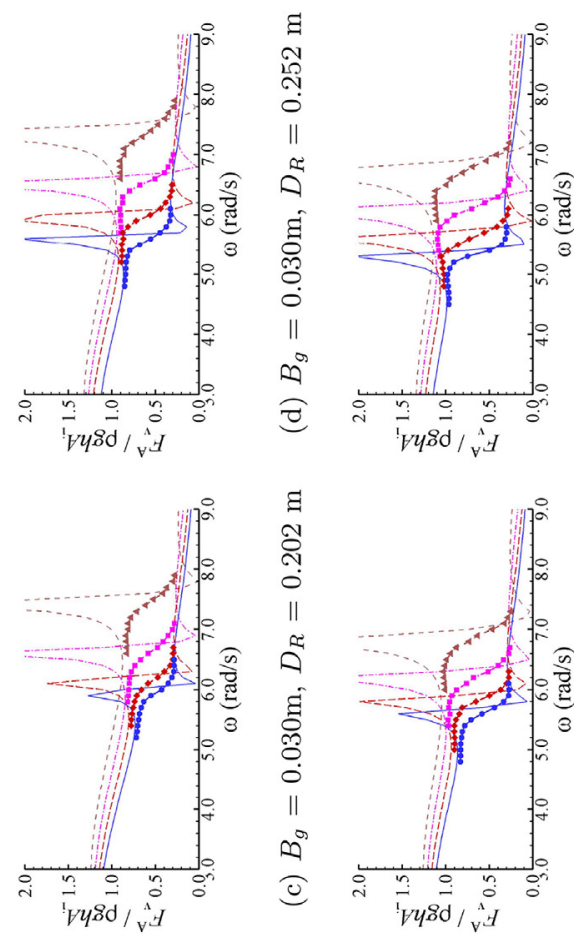

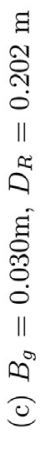

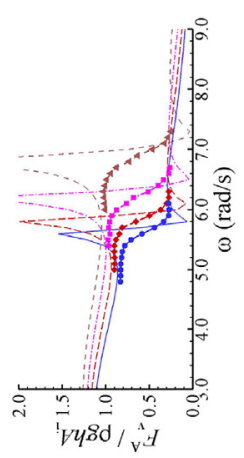

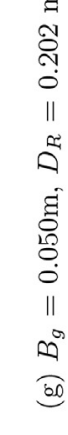

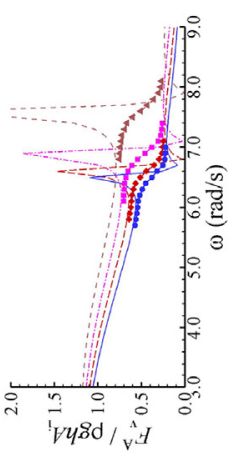

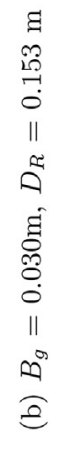
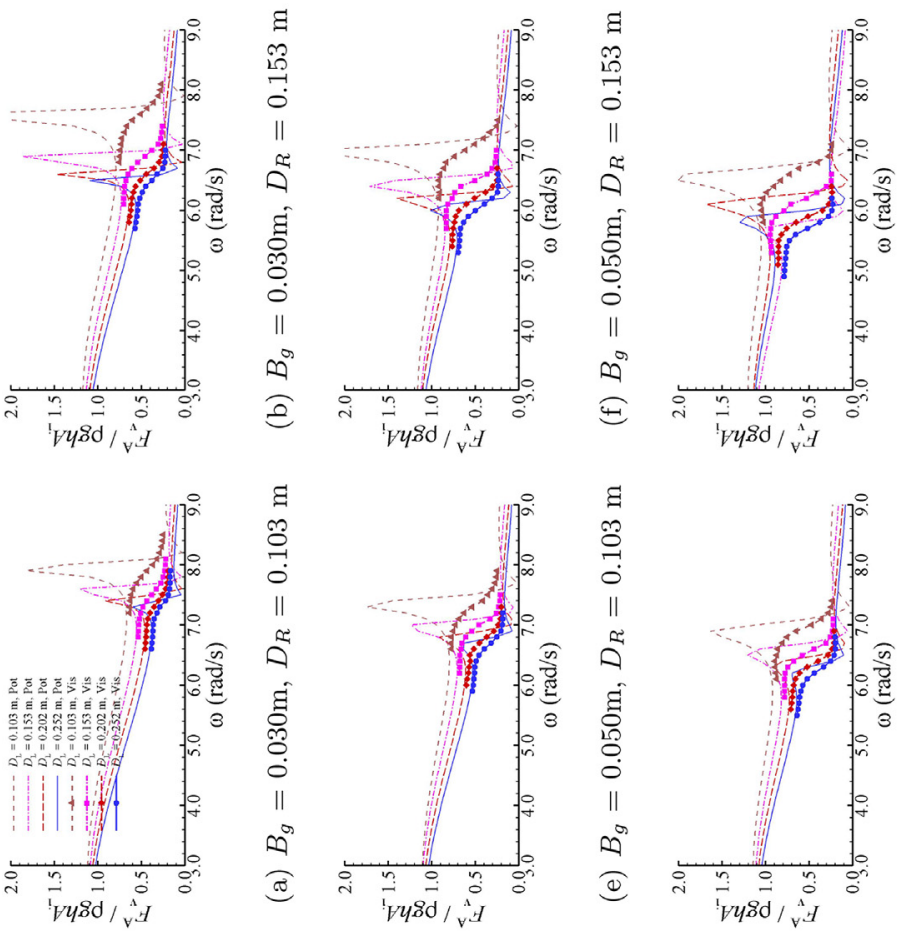

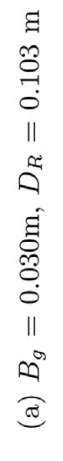
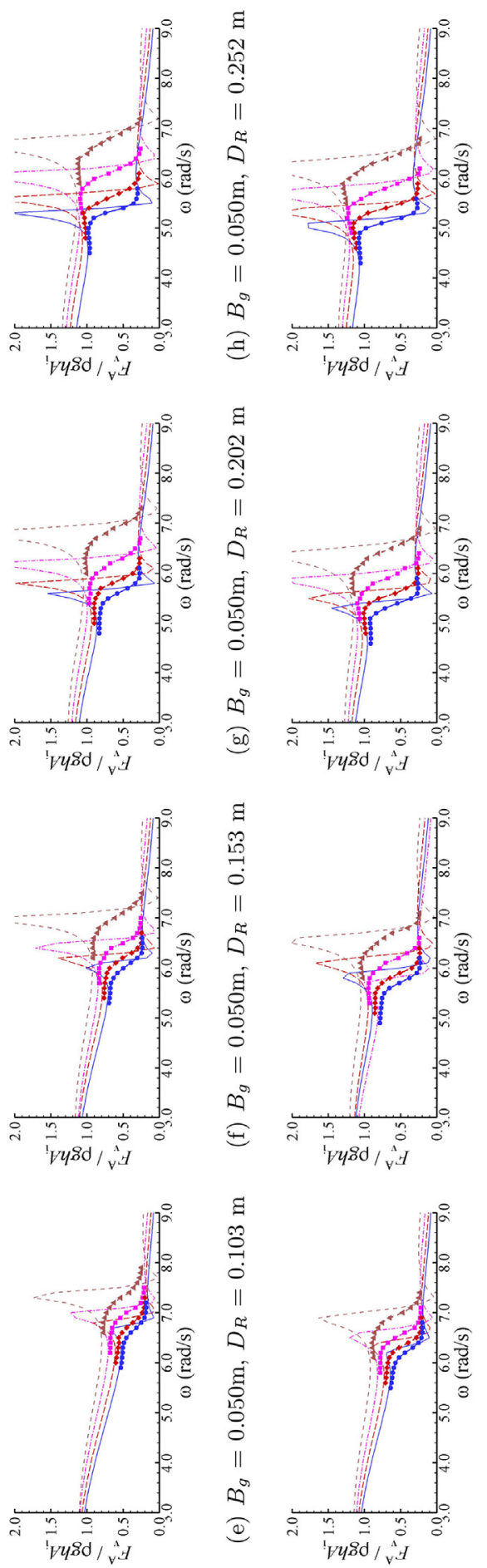
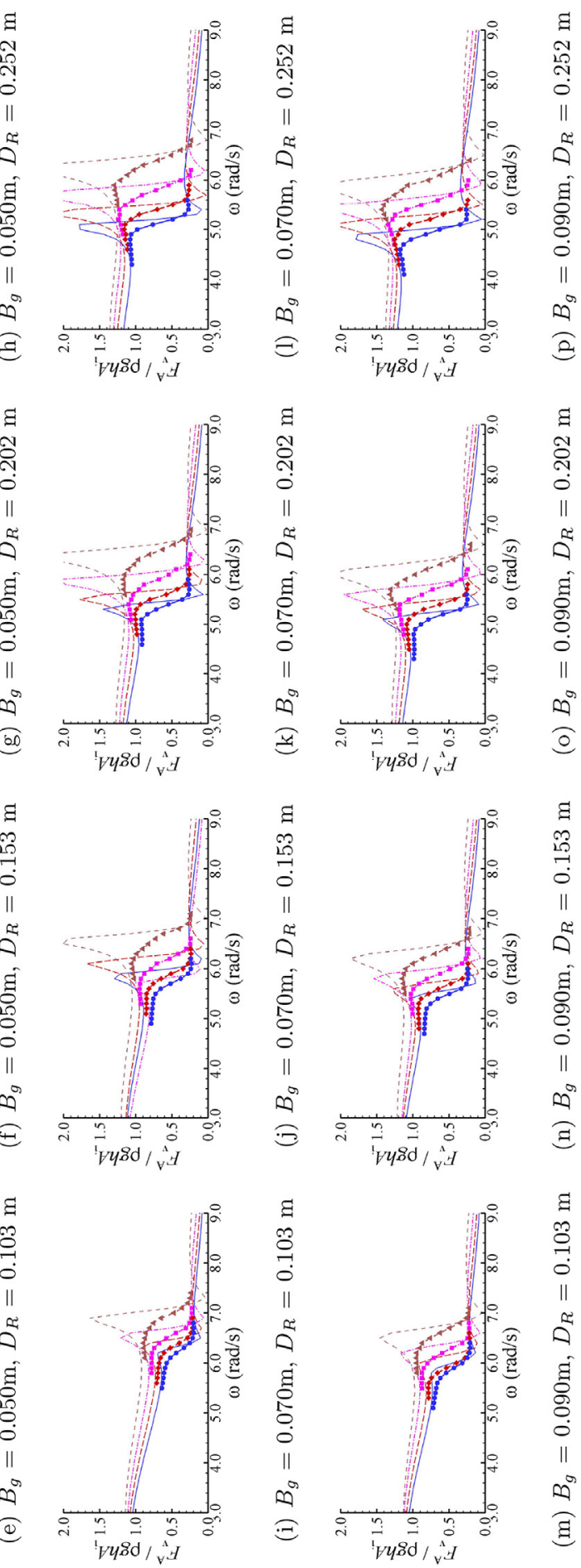

Fig. 16. Comparison of normalized vertical wave forces $F_{v}^{A}$ on Box A for various upstream box drafts $D_{L}$ under wave actions.

be observed, which is dependent on the downstream box drafts $D_{R}$. When $D_{R}$ is smaller than $D_{L}$, Box B is shielded by Box A. In this situation, the increase of upstream box draft $D_{L}$ causes a stronger shielding effect on the wave forces on Box B. A quintessential example could be cited in the first column of Figs. 15 and 17, where the draft of Box B is the smallest one, $D_{R}=0.103 \mathrm{~m}$. It can be observed that the magnitudes of $F_{h}^{B}$ and $F_{v}^{B}$ decrease with the increase of upstream box drafts. When $D_{R}$ is larger than $D_{L}$, Box B and the narrow gap between two boxes are exposed to the incident wave action. In this situation, the complex hydrodynamic behavior in the vicinity of narrow gap can be captured during the large-amplitude piston-like free surface oscillation, including the vortex 

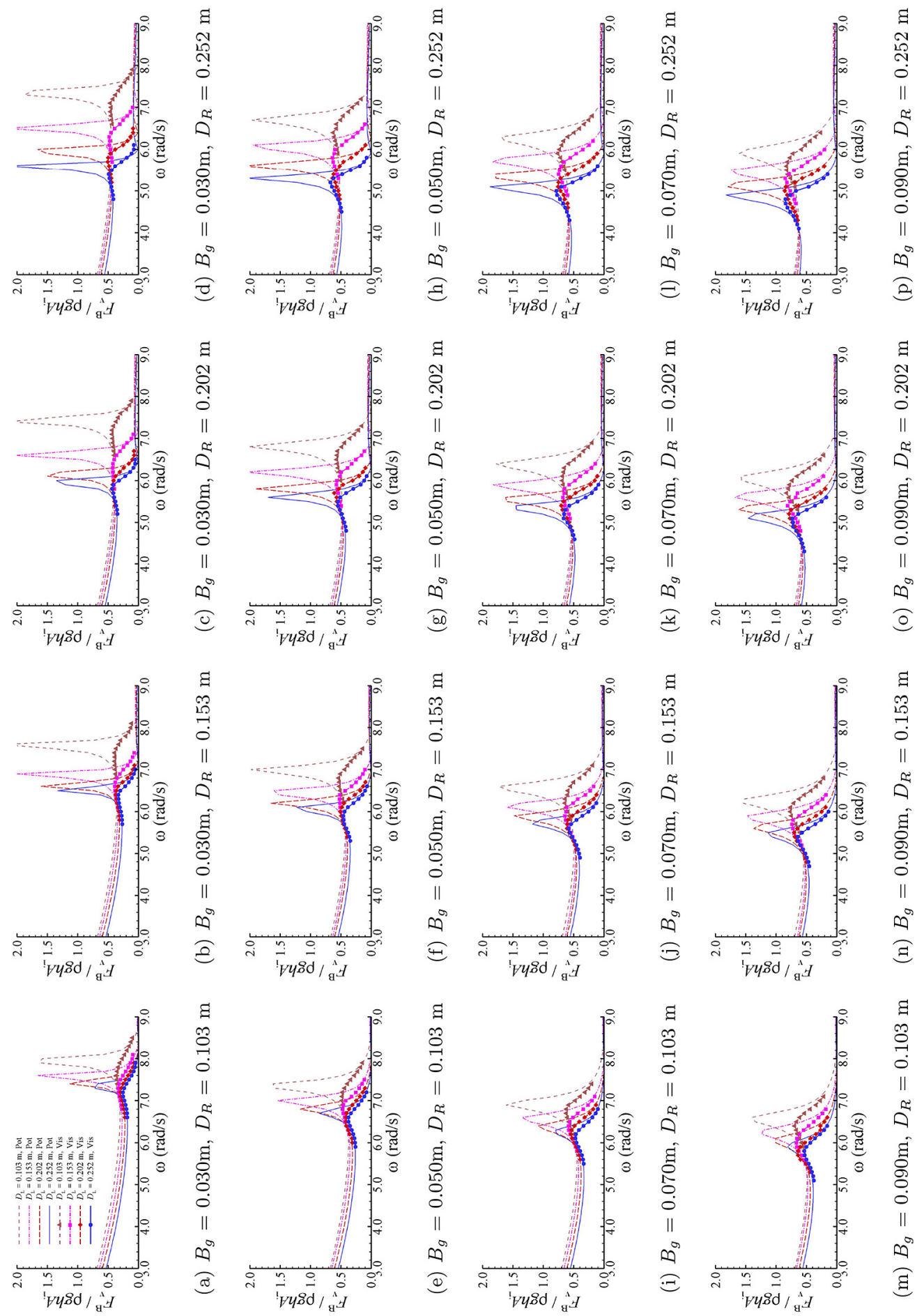

Fig. 17. Comparison of normalized vertical wave forces $F_{v}^{B}$ on Box B for various upstream box drafts $D_{L}$ under wave actions.

shedding from the trailing sharp edge of upstream box, the wave reflection from the leading wall of downstream box, and the wave transformation below the bottom of downstream box. The hydrodynamic behavior mentioned above would lead to the complex dependence of $F_{h}^{B}$ and $F_{v}^{B}$ against the upstream box draft. A typical comparison can be observed in the fourth column of Figs. 15 and 17 , where the draft of Box B is the largest one, $D_{R}=0.252 \mathrm{~m}$. More complex characteristics of wave forces on Box B with various upstream box drafts can be observed in the second and third columns of Figs. 15 and 17. When the draft of downstream Box B is the medium one, the shield effect and the direct wave action discussed above are combined in this scenario. All these variations of wave 

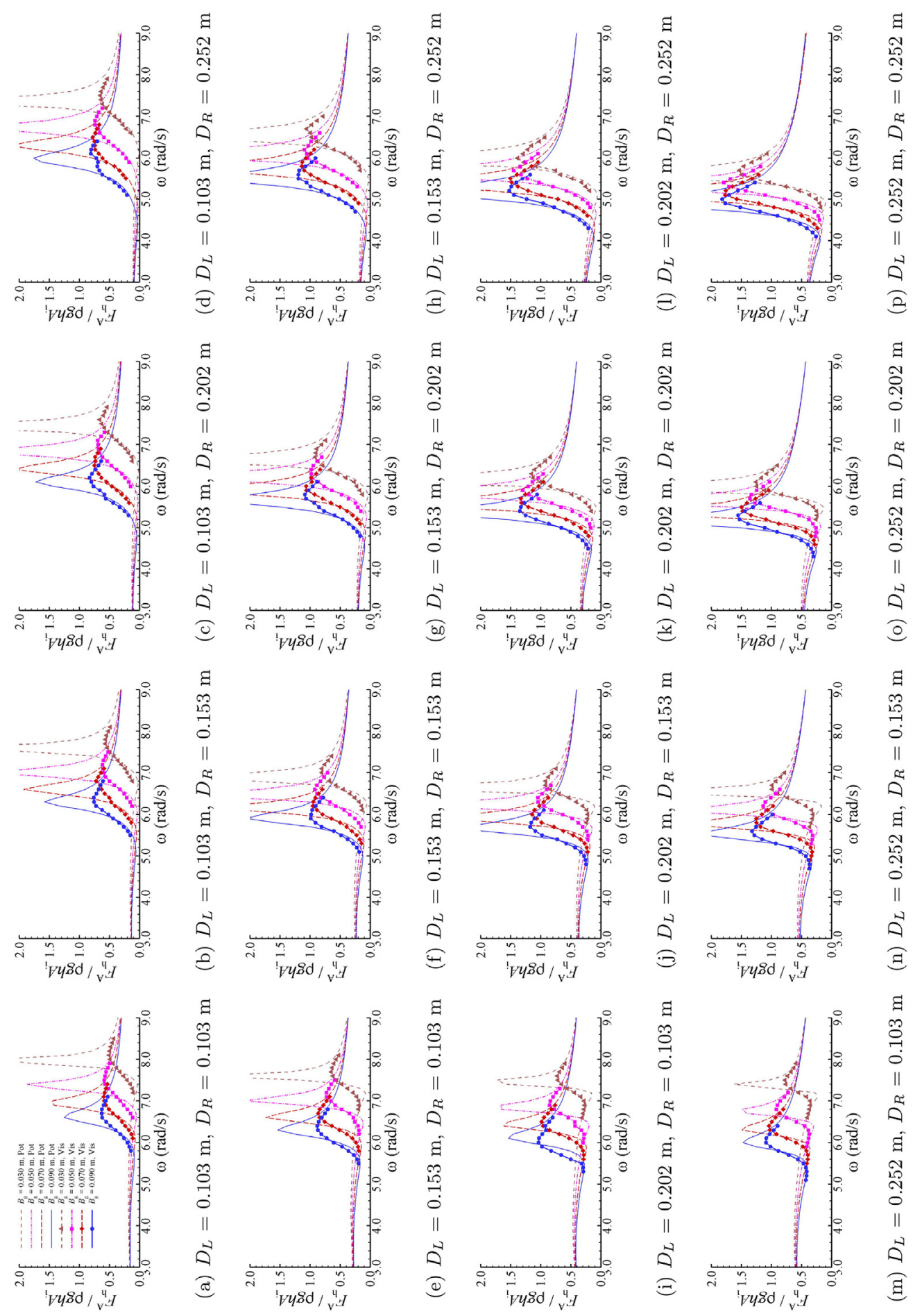

Fig. 18. Comparison of normalized horizontal wave forces $F_{h}^{A}$ on Box A for various gap breadth $B_{g}$ under wave actions.

forces on Box B $F_{h}^{B}$ and $F_{v}^{B}$ are mainly from the fluid oscillation in the narrow gap, and consequently similar hydrodynamic behavior can also be found in Ref. [11]. Finally, the variation of wave forces is significantly affected by the upstream box draft when a larger downstream box draft appears. 

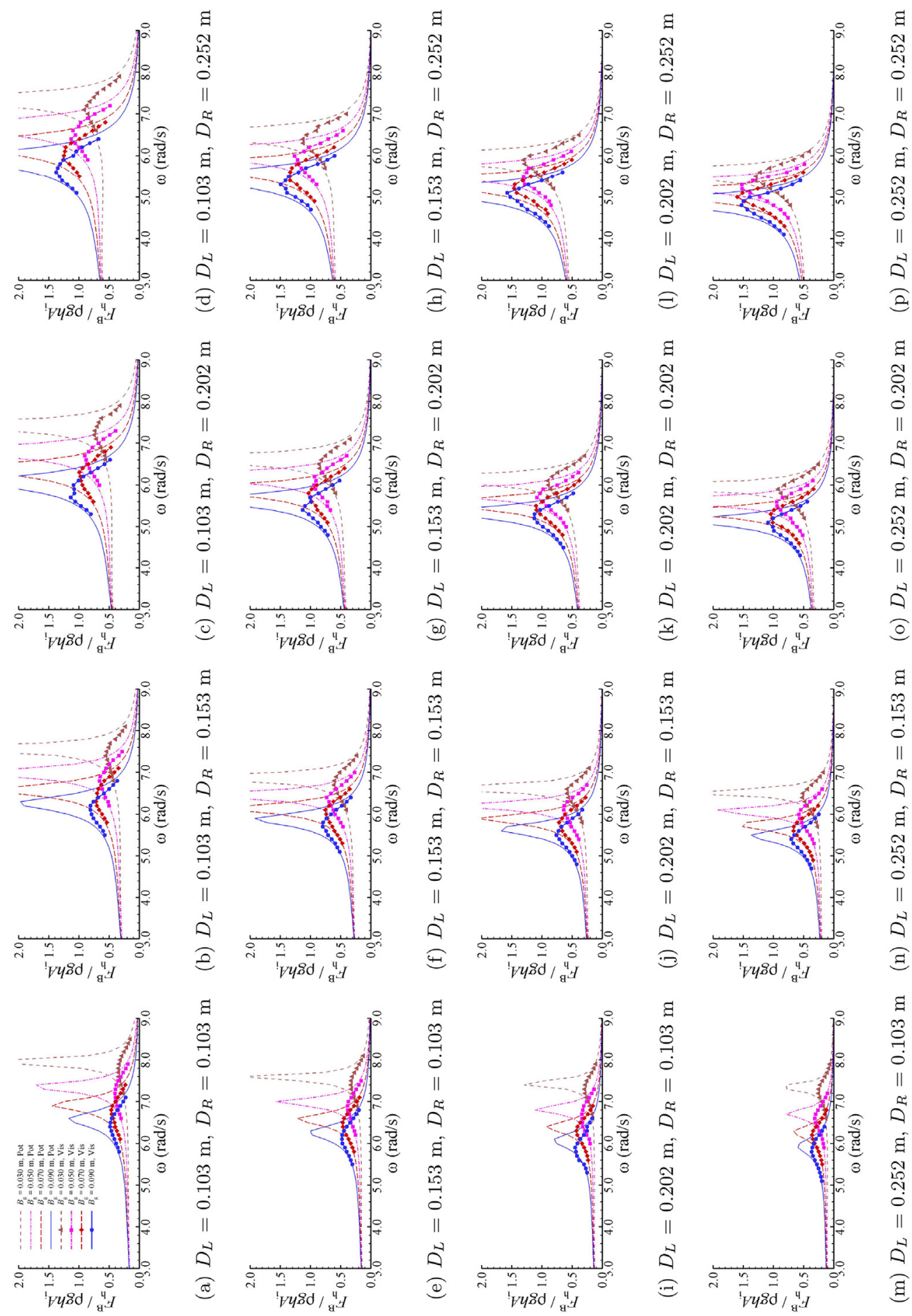

Fig. 19. Comparison of normalized horizontal wave forces $F_{h}^{B}$ on Box B for various gap breadth $B_{g}$ under wave actions.

\subsection{Influence of gap breadth}

The influence of gap breadth between two boxes on the variations of horizontal and vertical wave forces on Boxes A and B are investigated in Figs. 18-21. The insignificant effect of gap breadth on wave forces can be observed at the scopes of low and high frequencies. In the region of medium frequencies, the magnitudes of wave forces on Boxes A and B have close dependence on gap breadth, especially around resonant frequencies. A general comparison in Figs. 18 and 19 for horizontal wave forces on Boxes A and B 

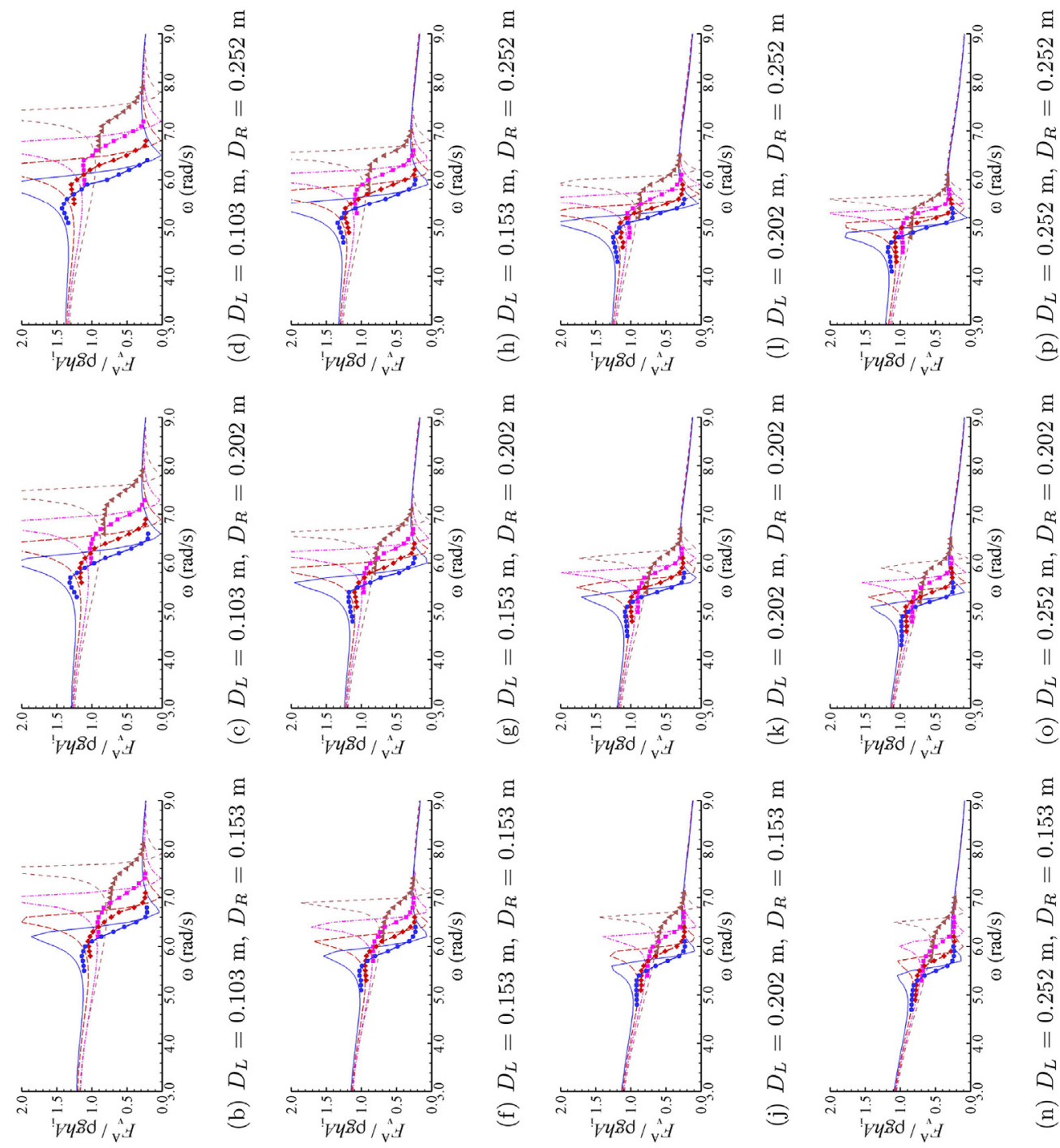

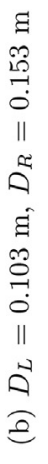
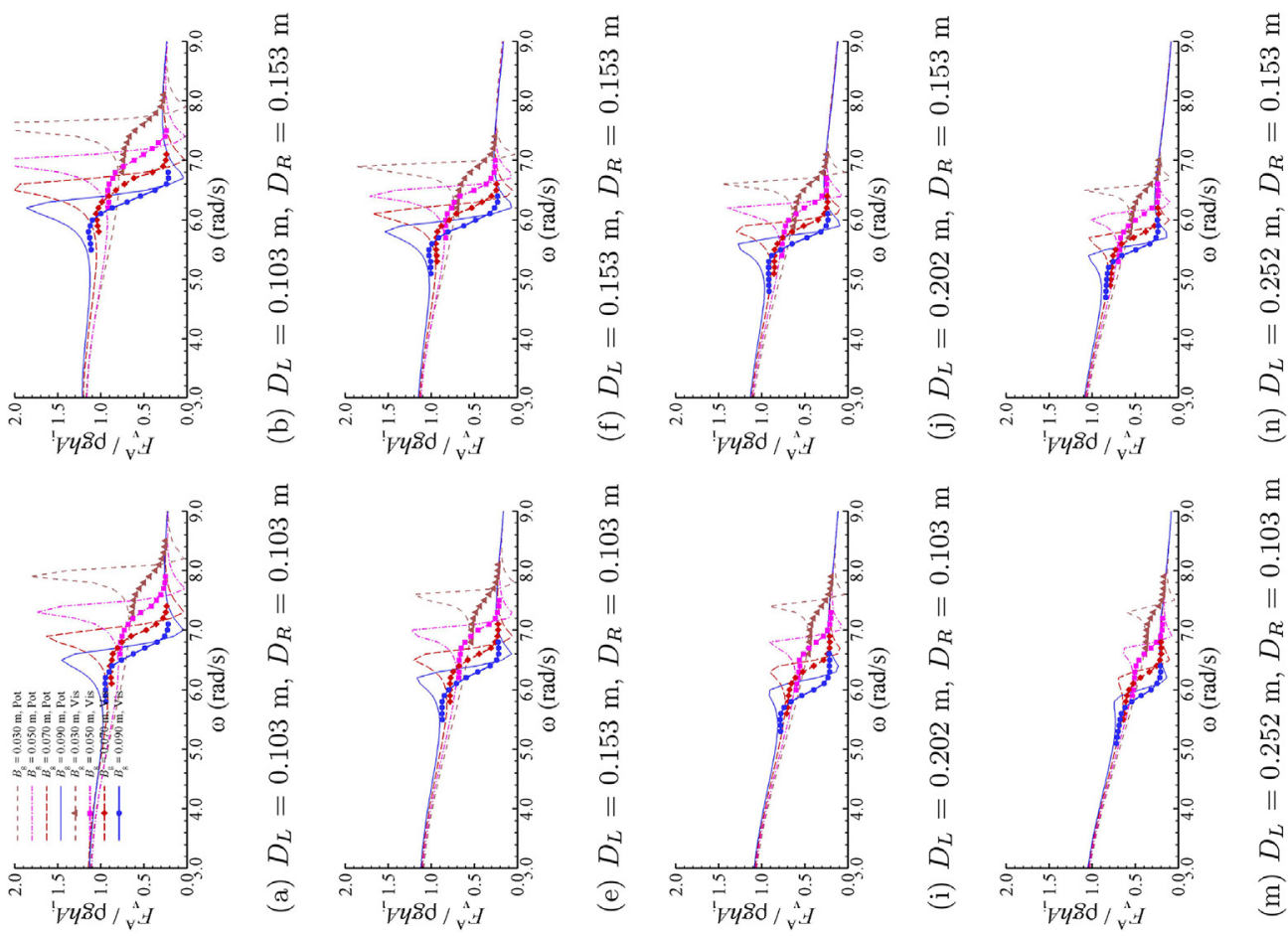

Fig. 20. Comparison of normalized vertical wave forces $F_{v}^{A}$ on Box A for various gap breadth $B_{g}$ under wave actions.

manifest that the increase of gap breadth can lead to smaller resonant frequencies and larger resonant horizontal wave forces. The percentages of increase (compared to the results for the gap breadth to be $90 \mathrm{~mm}$ ) in horizontal wave forces on Boxes A and B at resonant frequencies against the gap breadth varying $30 \mathrm{~mm}-90 \mathrm{~mm}$ are tabulated in Table 3 . It can be observed that the largest percentages occur in the case of DL202DR103, which are $43.7 \%$ and $54.7 \%$ for $F_{h}^{A}$ and $F_{h}^{B}$, respectively. A general estimation of 1.1-1.5 times force increase by tripling the gap breadth can be suggested at the scope of present numerical simulations. When the incident wave frequencies $\omega$ are smaller than resonant frequencies, the horizontal wave forces $F_{h}^{A}$ and $F_{h}^{B}$ increase with the increase of 

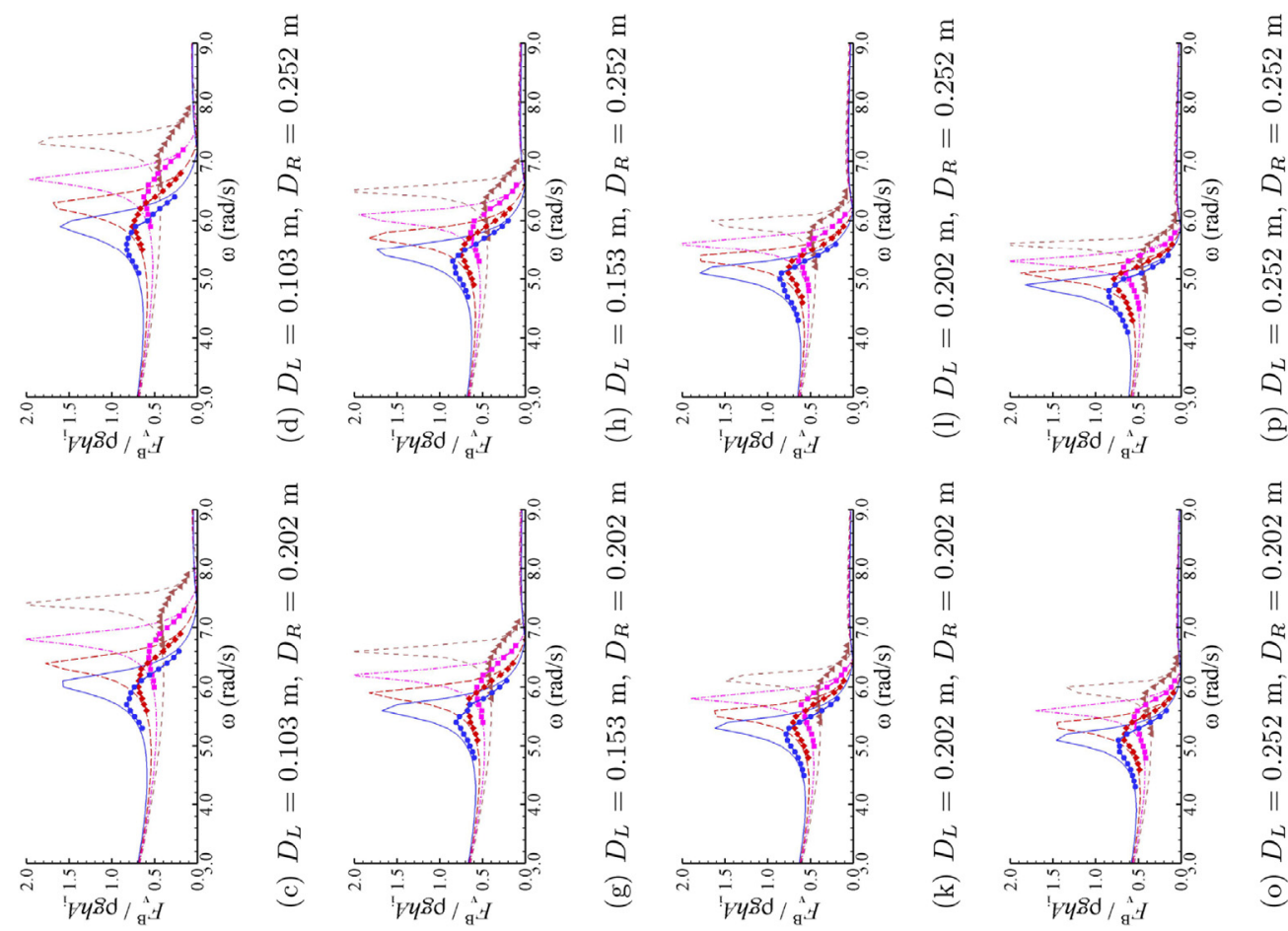

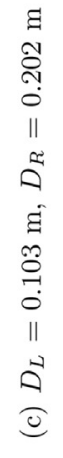
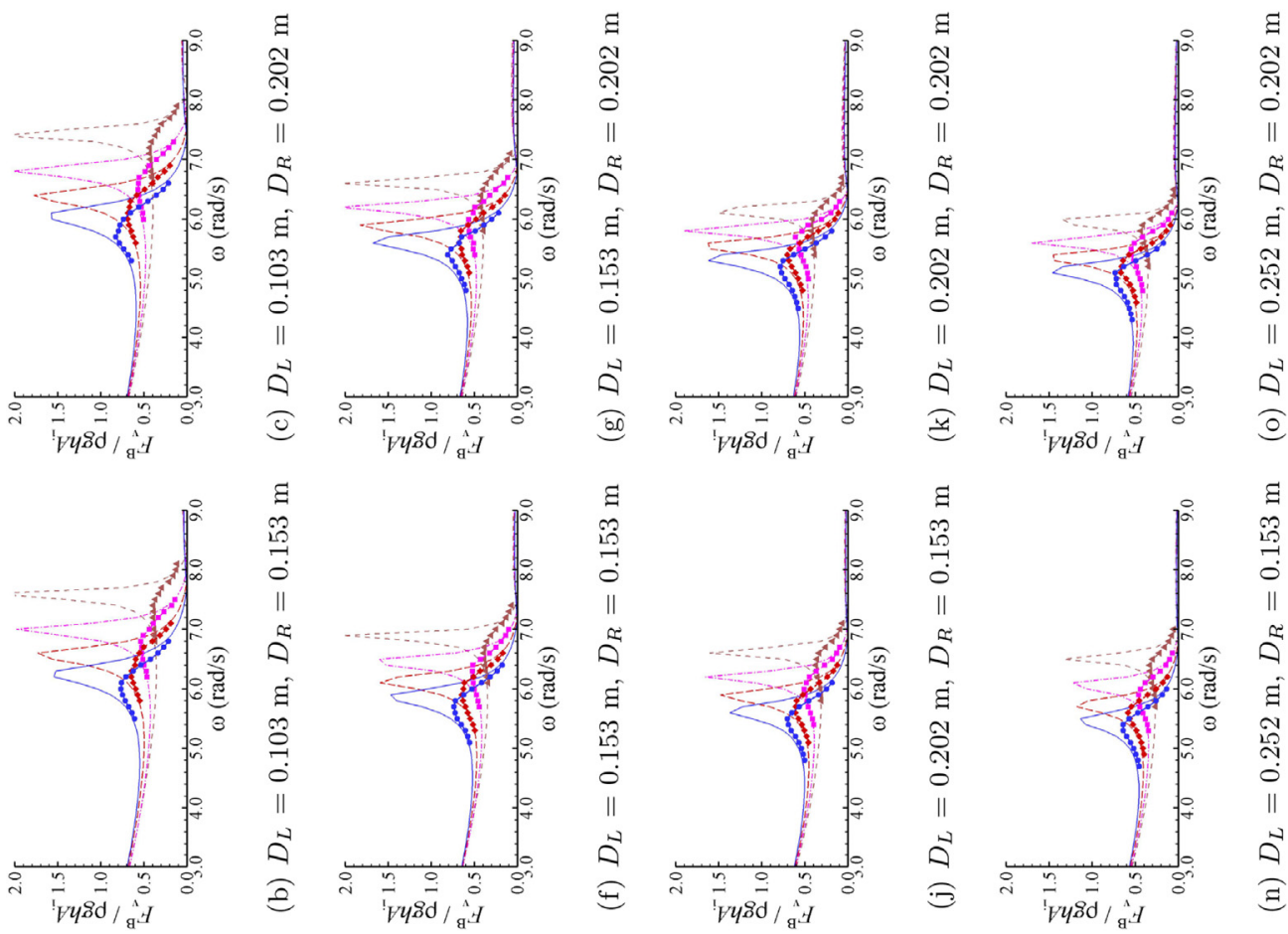

$a$
0
03
0
0
$\| 1$
0
0
a
0
0
0
11
0
0
0
0
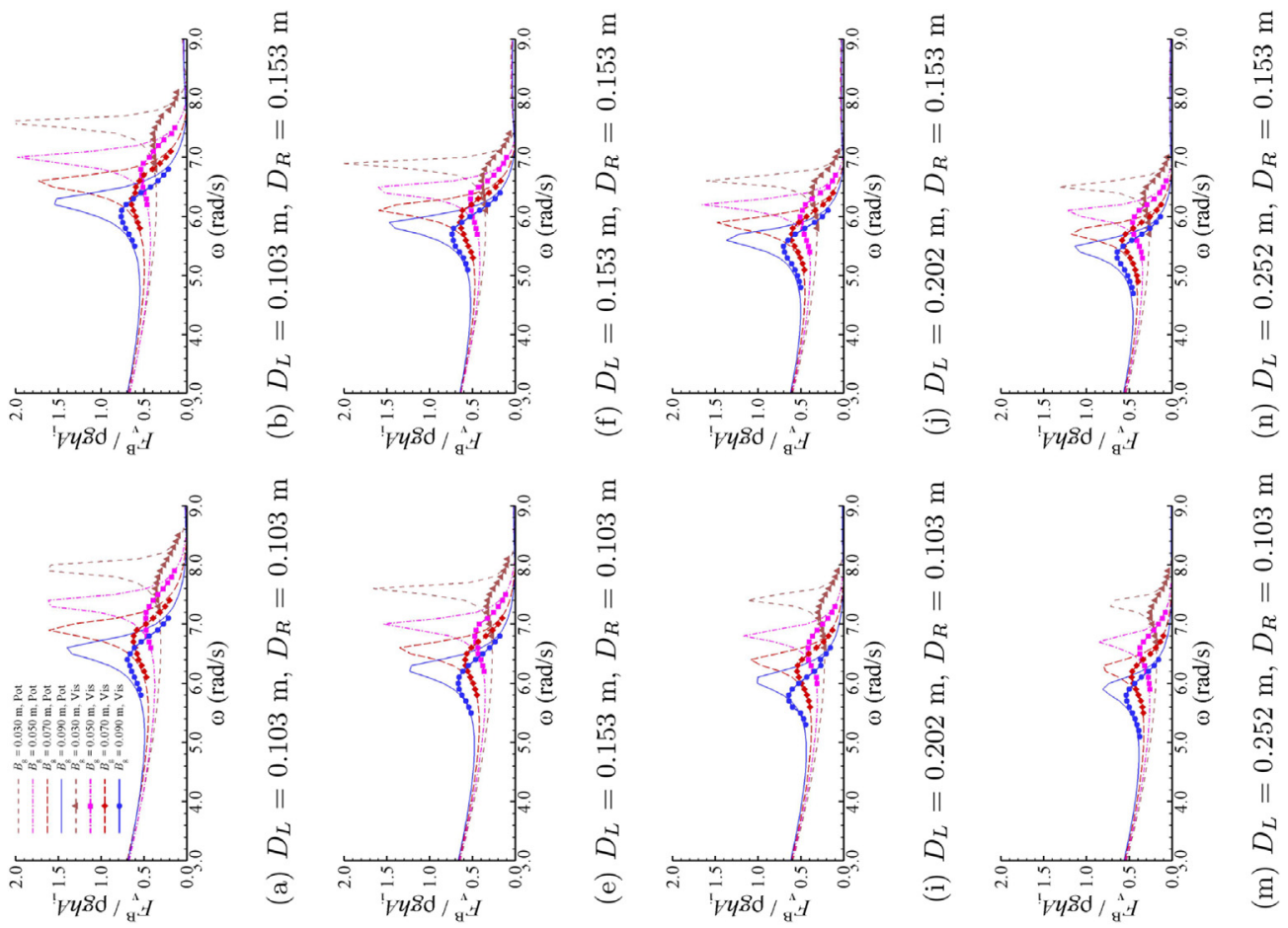

Fig. 21. Comparison of normalized vertical wave forces $F_{v}^{B}$ on Box B for various gap breadth $B_{g}$ under wave actions.

gap breadth. In contrast, the smaller $F_{h}^{A}$ and $F_{h}^{B}$ can be observed for the larger gap breadth when $\omega$ are larger than resonant frequencies.

Figs. 20 and 21 indicate the influence of gap breadth between two boxes on the vertical wave forces $F_{v}^{A}$ and $F_{v}^{B}$ on Boxes A and B with incident wave frequencies $\omega$. The magnitudes of vertical wave forces $F_{v}^{A}$ on Box A decrease gradually with incident wave frequencies $\omega$, and then decrease rapidly at some characteristic frequencies. At these scopes of frequencies, the increase of gap breadth can give rise to the increase of wave forces and the decrease of the characteristic frequencies. When the incident wave 
Table 3

The percentages of increase in resonant horizontal wave forces on Boxes A and B for the gap breadth from $30 \mathrm{~mm}$ to $90 \mathrm{~mm}$.

\begin{tabular}{|c|c|c|c|c|}
\hline$\left(F_{h}^{A}, F_{h}^{B}\right)$ & $D_{R}=0.103 \mathrm{~m}$ & $D_{R}=0.153 \mathrm{~m}$ & $D_{R}=0.202 \mathrm{~m}$ & $D_{R}=0.252 \mathrm{~m}$ \\
\hline$D_{L}=0.103 \mathrm{~m}$ & $25.2 \%, 42.0 \%$ & $33.0 \%, 46.7 \%$ & $25.8 \%, 52.9 \%$ & $8.1 \%, 51.4 \%$ \\
\hline$D_{L}=0.153 \mathrm{~m}$ & $36.6 \%, 46.6 \%$ & $22.9 \%, 30.7 \%$ & $18.9 \%, 32.5 \%$ & $12.8 \%, 46.4 \%$ \\
\hline$D_{L}=0.202 \mathrm{~m}$ & $43.7 \%, 54.7 \%$ & $27.6 \%, 30.6 \%$ & $20.0 \%, 25.2 \%$ & $10.1 \%, 21.1 \%$ \\
\hline$D_{L}=0.252 \mathrm{~m}$ & $41.3 \%, 46.5 \%$ & $33.8 \%, 35.9 \%$ & $24.0 \%, 18.5 \%$ & $18.6 \%, 18.7 \%$ \\
\hline
\end{tabular}

frequencies are larger than the characteristic frequencies, the decrease of wave forces with the increase of gap breadth can be observed in Fig. 20. As for the vertical wave forces $F_{v}^{B}$ on Box B, the maximal wave forces can be obtained at some particular wave frequencies, which are resonant frequencies of wave forces. The resonant frequencies tend to decrease with the increase of gap breadth and the corresponding resonant amplitudes of wave forces increase. Table 4 shows that the largest increase in the vertical force increase can approach more than two times by tripling the gap breadth in the present study. When the incident wave frequencies are smaller or larger than resonant frequencies, the magnitudes of wave forces are larger or smaller for larger gap breadth, respectively.

\subsection{Influence of incident wave amplitude}

The influence of incident wave amplitude on the horizontal and vertical wave forces on Boxes A and B is investigated in Figs. 22-25. Three incident wave amplitudes, $A_{i}=0.008 \mathrm{~m}, 0.012 \mathrm{~m}$, and $0.016 \mathrm{~m}$ are adopted for numerical simulations, in which the gap breadth $B_{g}=0.050 \mathrm{~m}$ with various box drafts is employed. Again, the linear potential solutions are included for the purpose of comparison in these figures.

The magnitudes of horizontal and vertical wave forces on Boxes A with different incident wave amplitudes are depicted in Figs. 22 and 24. A two-phase variation at the scope of medium frequencies can be observed for the normalized horizontal and vertical wave forces $F_{h}^{A} / \rho g h A_{i}$ and $F_{v}^{A} / \rho g h A_{i}$. As shown in Fig. 22, the larger incident wave amplitude is able to generate the larger $F_{h}^{A} / \rho g h A_{i}$ at the lower scope of medium frequencies. With the increase of incident wave frequency, the resonant amplitudes of $F_{h}^{A} / \rho g h A_{i}$ and corresponding resonant frequencies can be observed, in which $F_{h}^{A} / \rho g h A_{i}$ decrease significantly with the increase of incident wave amplitude. The resonant frequencies of $F_{h}^{A} / \rho g h A_{i}$ slightly increase with the increase of incident wave amplitude, although the opposite situation can be observed for the wave response in the narrow gap [11]. For example, the resonant frequency of wave force on Box A varies from $\omega=6.10 \mathrm{rad} / \mathrm{s}$ to $6.20 \mathrm{rad} / \mathrm{s}$ when the incident wave amplitude is changed from $A_{i}=0.008 \mathrm{~m}$ to $0.016 \mathrm{~m}$ for the case of Bg50DL153DR252. As for the vertical wave forces on Box A, Fig. 24 manifests the decrease and increase of normalized wave forces $F_{v}^{A} / \rho g h A_{i}$ with the increase of incident wave amplitude in the lower and higher regions of medium frequencies, respectively. All these hydrodynamic behaviors of wave forces on Box A have some analogous with the wave response in the narrow gap in Ref. [11]; but the evident discrepancy between them can also be found according to the analysis above. The large-amplitude piston-type of free surface oscillation in the narrow gap is an important factor for the wave forces around resonant frequencies. Meanwhile, the wave reflection causes the large-amplitude wave response in the front of Box A, which also has the significant effect on the wave forces. Those two factors lead to the similarity and discrepancy between the wave response in the narrow gap and the wave forces on Box A. When the incident wave frequencies are far from the resonant frequencies, $F_{h}^{A} / \rho g h A_{i}$ and $F_{v}^{A} / \rho g h A_{i}$ have little dependence on the incident wave amplitude.

Figs. 23 and 25 illustrate the influence of incident wave amplitude on the horizontal and vertical wave forces on Box B. The variations of normalized horizontal and vertical wave forces, $F_{h}^{B} / \rho g h A_{i}$ and $F_{v}^{B} / \rho g h A_{i}$, with three incident wave amplitudes are examined. Numerical simulations suggest that the incident wave amplitude has little effect on the normalized wave forces when the incident frequencies are outside a certain band from resonant frequencies. Around resonant frequencies, $F_{h}^{B} / \rho g h A_{i}$ and $F_{v}^{B} / \rho g h A_{i}$ decrease significantly with the increase of incident wave amplitude. Slight decrease of the resonant frequencies of $F_{h}^{B} / \rho g h A_{i}$ and $F_{v}^{B} / \rho g h A_{i}$ with the increase of incident wave amplitude can be observed in these figures. The above variations are almost the same with the wave response in the narrow gap in Ref. [11]. In difference to the wave forces on Box A, the wave response behind Box B is mild due to the shielding effect of two-boxes. The free surface in the narrow gap correspondingly becomes the determinant factor for wave forces on Box B, which results in the analogous between the wave response in the narrow gap and the wave force on Box $\mathrm{B}$ mentioned above.

Table 4

The increase percentage of increase in resonant vertical wave force on Box B for the gap breadth from $30 \mathrm{~mm}$ to $90 \mathrm{~mm}$.

\begin{tabular}{lllll}
\hline & $D_{R}=0.103 \mathrm{~m}$ & $D_{R}=0.153 \mathrm{~m}$ & $D_{R}=0.202 \mathrm{~m}$ & $D_{R}=0.252 \mathrm{~m}$ \\
\hline$D_{L}=0.103 \mathrm{~m}$ & $92.1 \%$ & $95.0 \%$ & $91.1 \%$ & $78.3 \%$ \\
$D_{L}=0.153 \mathrm{~m}$ & $102.2 \%$ & $89.6 \%$ & $89.8 \%$ & $76.0 \%$ \\
$D_{L}=0.202 \mathrm{~m}$ & $117.7 \%$ & $88.9 \%$ & $84.2 \%$ & $70.0 \%$ \\
$D_{L}=0.252 \mathrm{~m}$ & $111.3 \%$ & $94.2 \%$ & $69.3 \%$ & $72.3 \%$ \\
\hline
\end{tabular}




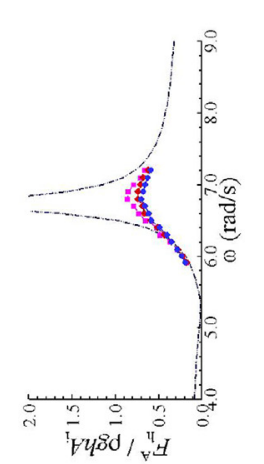

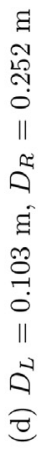

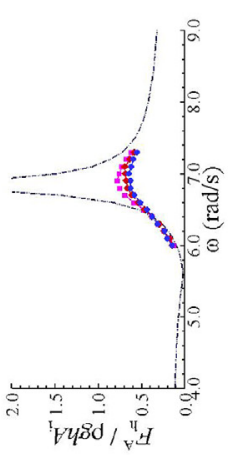

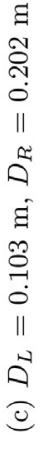

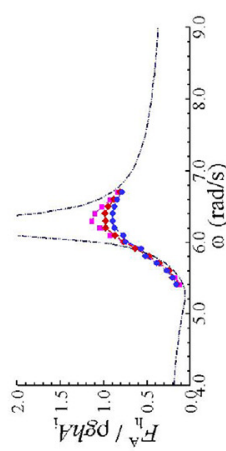

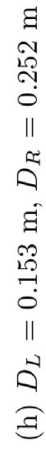

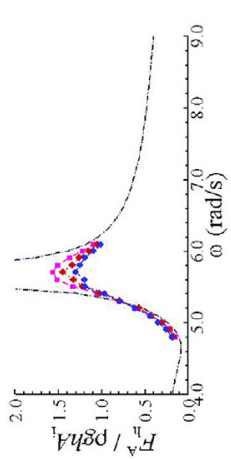

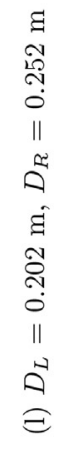
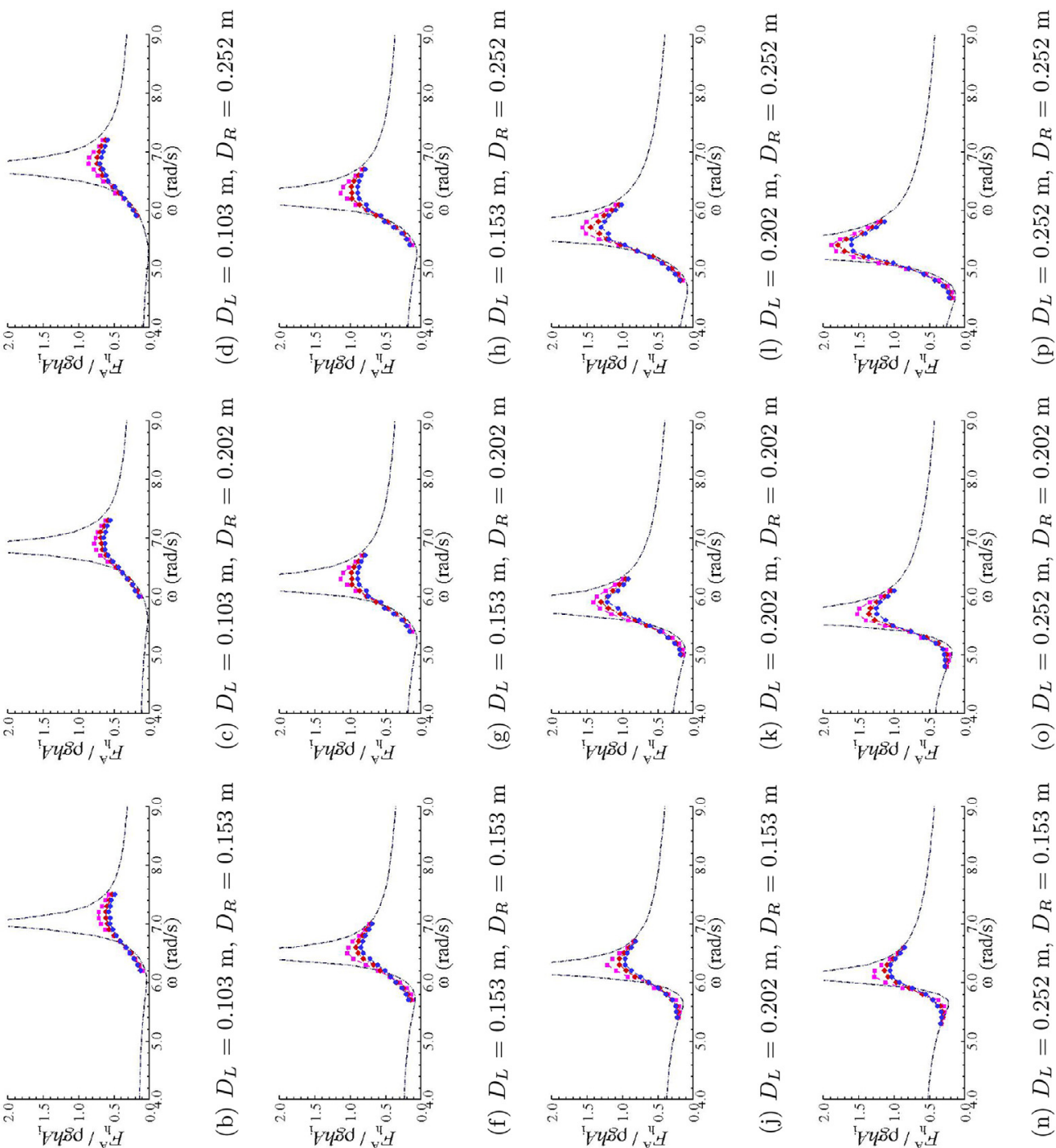

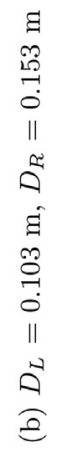

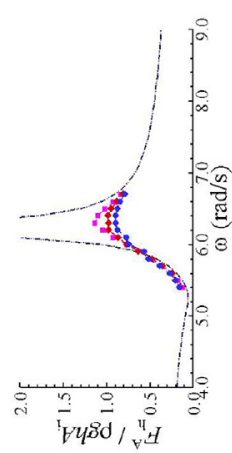

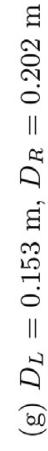

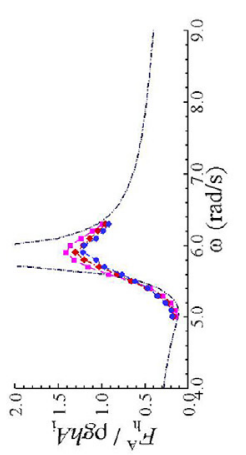

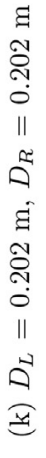
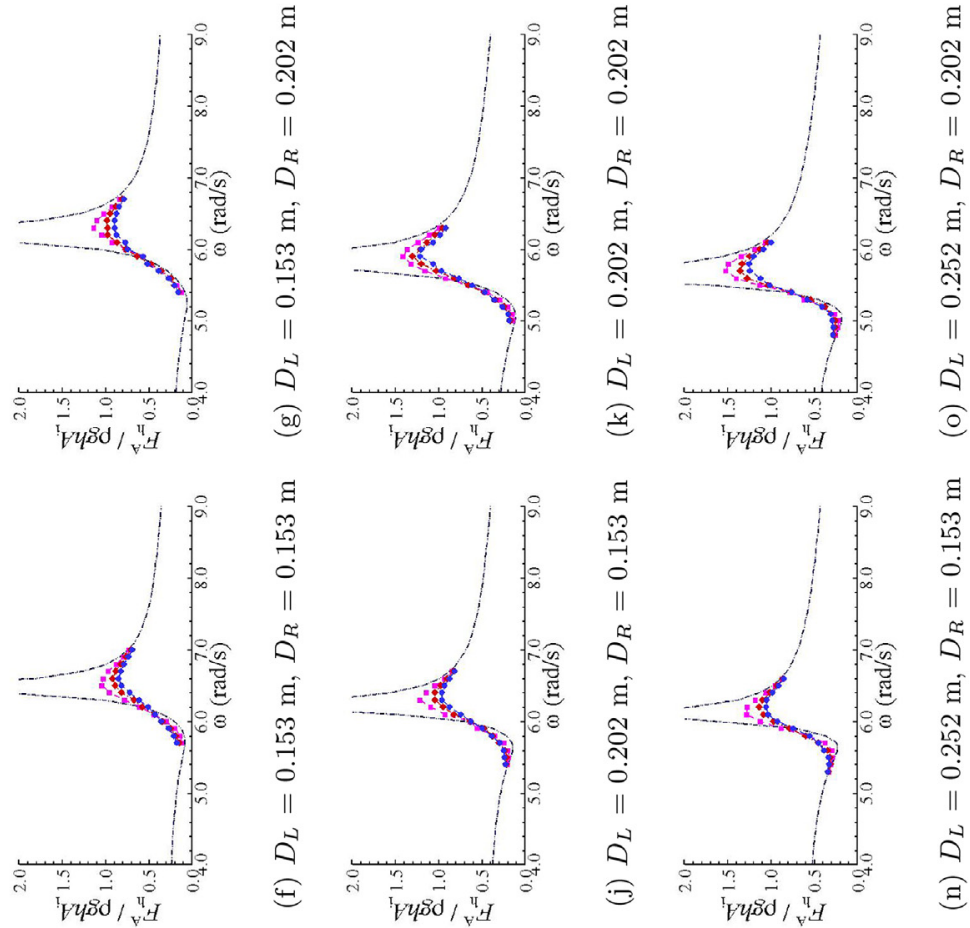

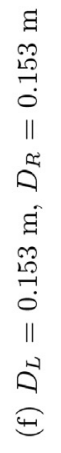

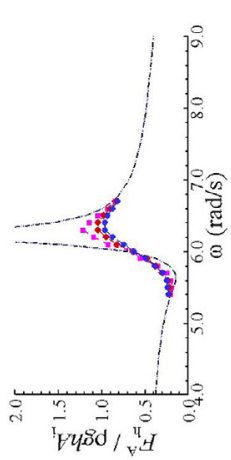

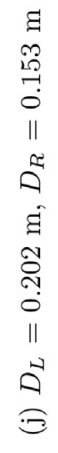
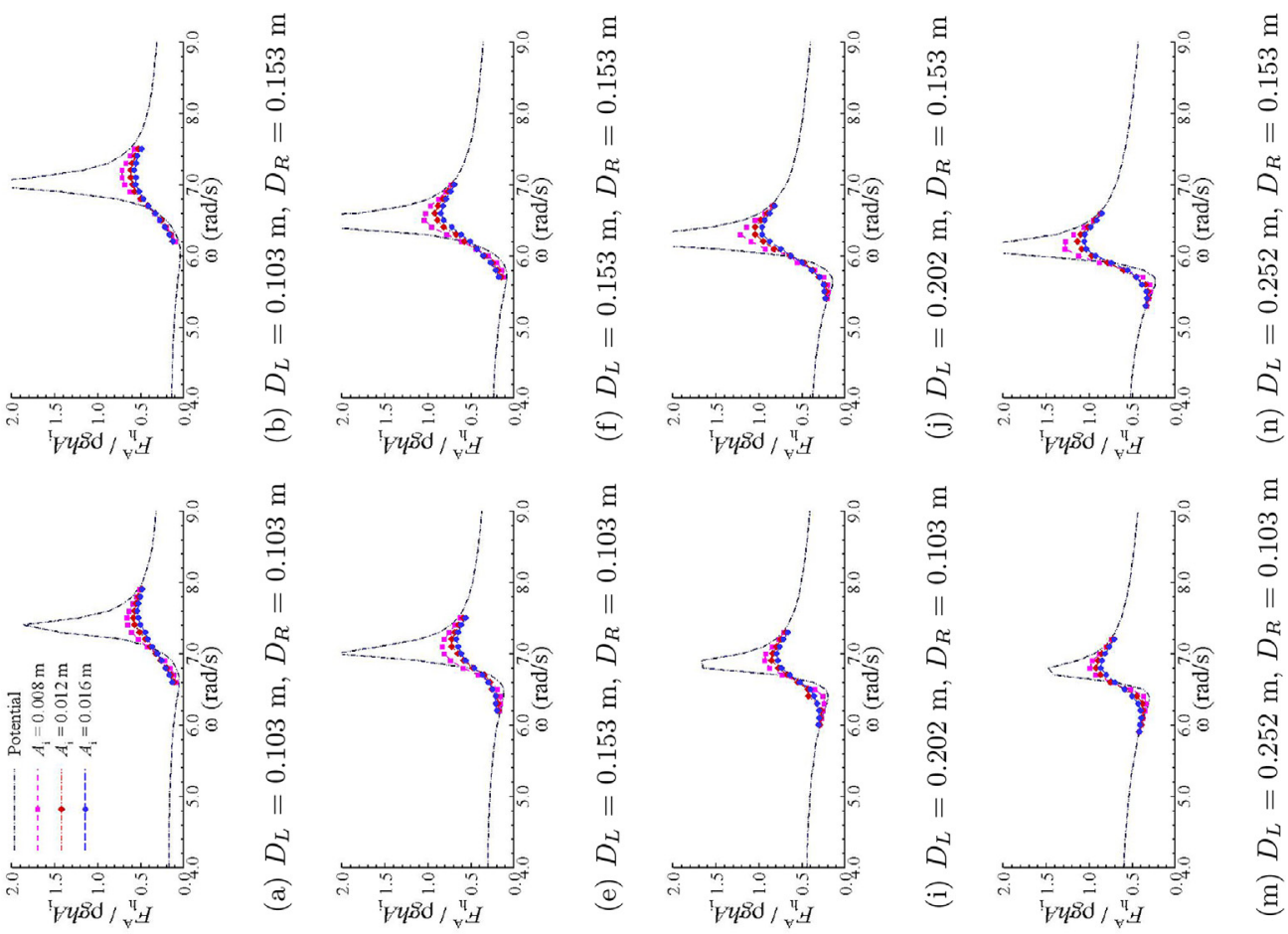

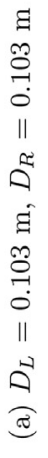
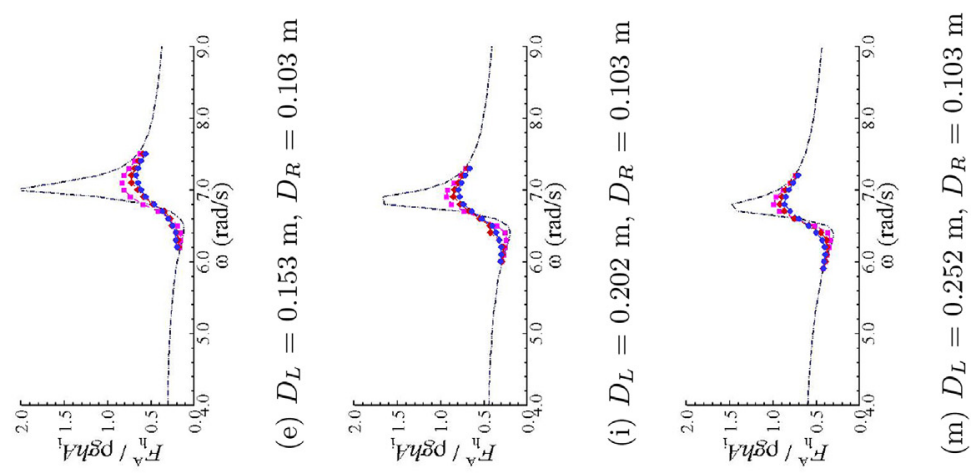

Fig. 22. Comparison of normalized horizontal wave forces $F_{h}^{A}$ on Box A under various incident wave amplitudes.

\section{Conclusion}

The main motivation behind this work is to investigate the similarity and discrepancy of the behavior between the wave response in the narrow gap and the wave forces on the side-by-side non-identical boxes in close proximity. This work is carried out by both viscous fluid flow and potential flow models for demonstrating the necessity of considering the fluid viscosity in this gap resonance problem. Numerical simulations suggest that the magnitudes of wave forces on two boxes present the three-phase variation with the 

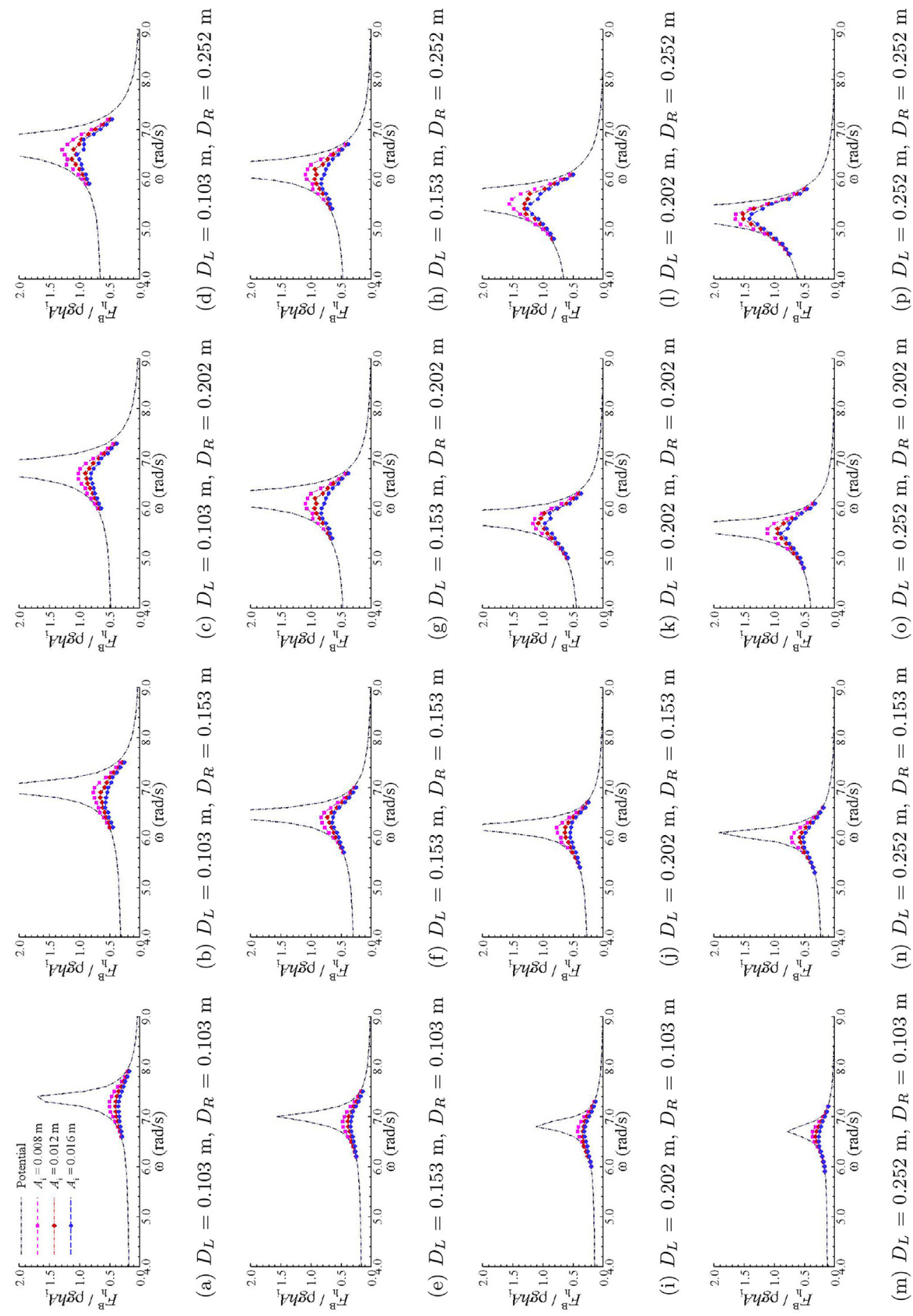

Fig. 23. Comparison of normalized horizontal wave forces $F_{h}^{B}$ on Box B under various incident wave amplitudes.

incident wave frequency, that is, low frequencies range, medium frequencies range, and high frequencies range. The inherent neglect of vortical flow and eddy motion in the linear potential model can lead to the over-prediction of wave forces in the medium frequencies range, especially around the resonant frequency. The exaggerated wave forces always occur at resonant frequencies of fluid oscillation. However, the viscous fluid flow results manifest that the resonant frequencies of wave forces on Boxes A and B are larger and smaller than that of wave response in the narrow gap, respectively, which is due to the influence of wave responses outside the boxes. 

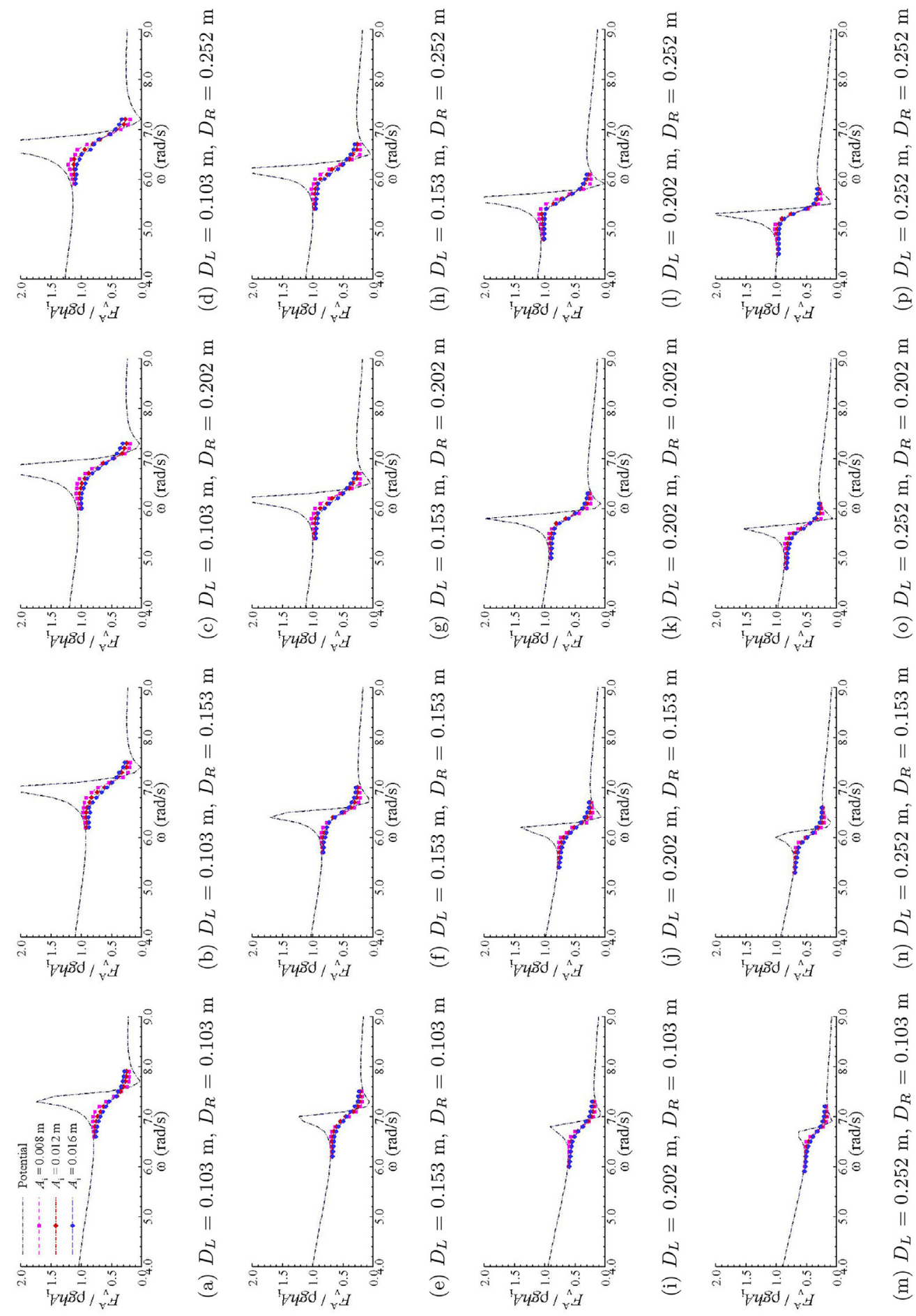

Fig. 24. Comparison of normalized vertical wave forces $F_{v}^{A}$ on Box A under various incident wave amplitudes.

The characteristic/resonant frequencies of wave forces on Boxes A and B tend to decrease with the increase of gap breadth, draft of upstream box and draft of downstream box. The configurations of two-box system have significant effect on the wave force on two boxes. Numerical simulations suggest that the total forces on the two boxes are smaller when the large draft box locates in the upstream, which can be recommended to the side-by-side operations. The variation of wave forces is more sensitivity to the downstream box draft when a larger upstream box draft appear. The same conclusion about the effect of upstream box draft can also be observed for a larger downstream box draft. Therefore, the behavior of wave forces should be paid more attention in the 

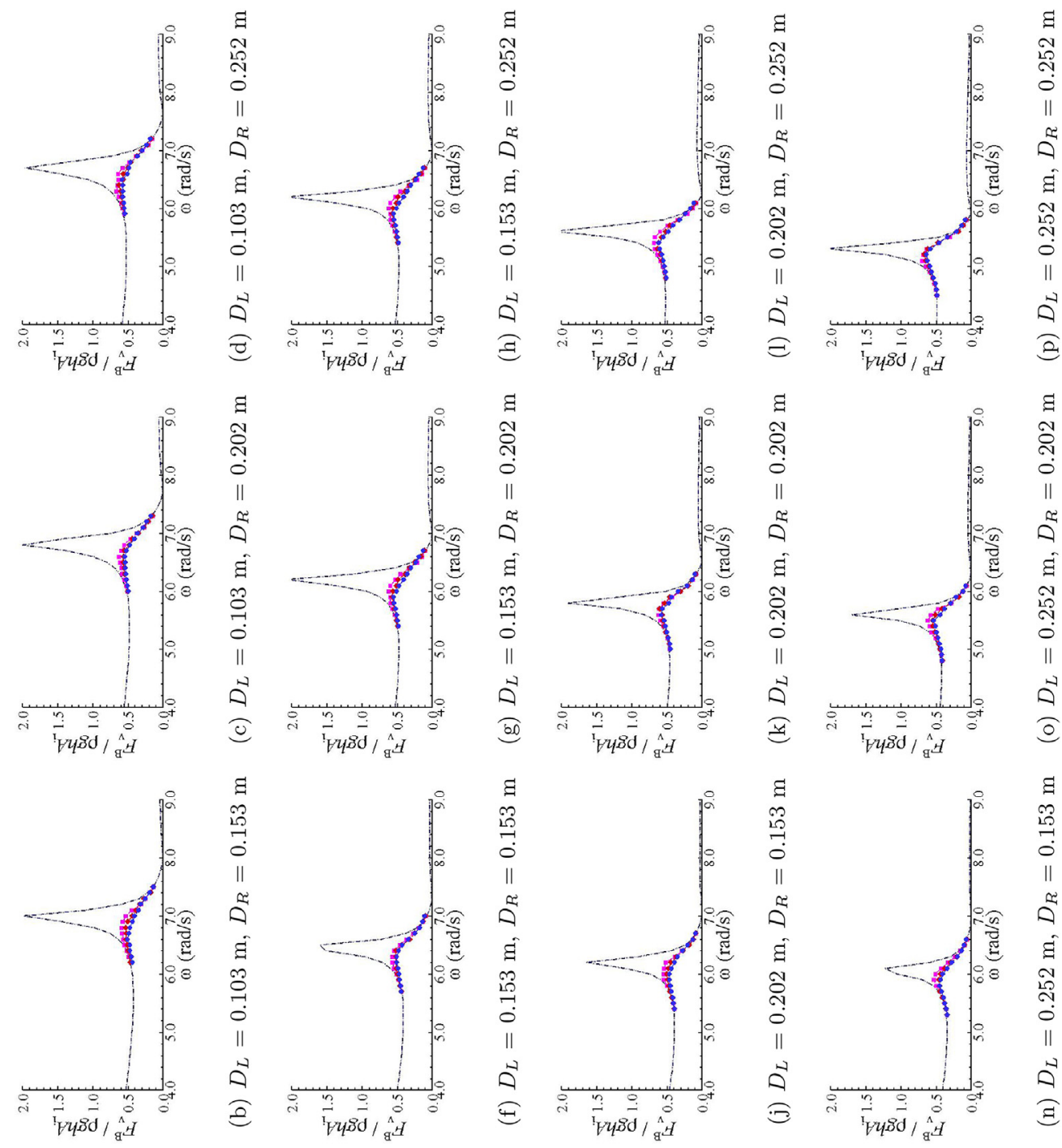

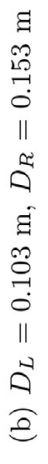
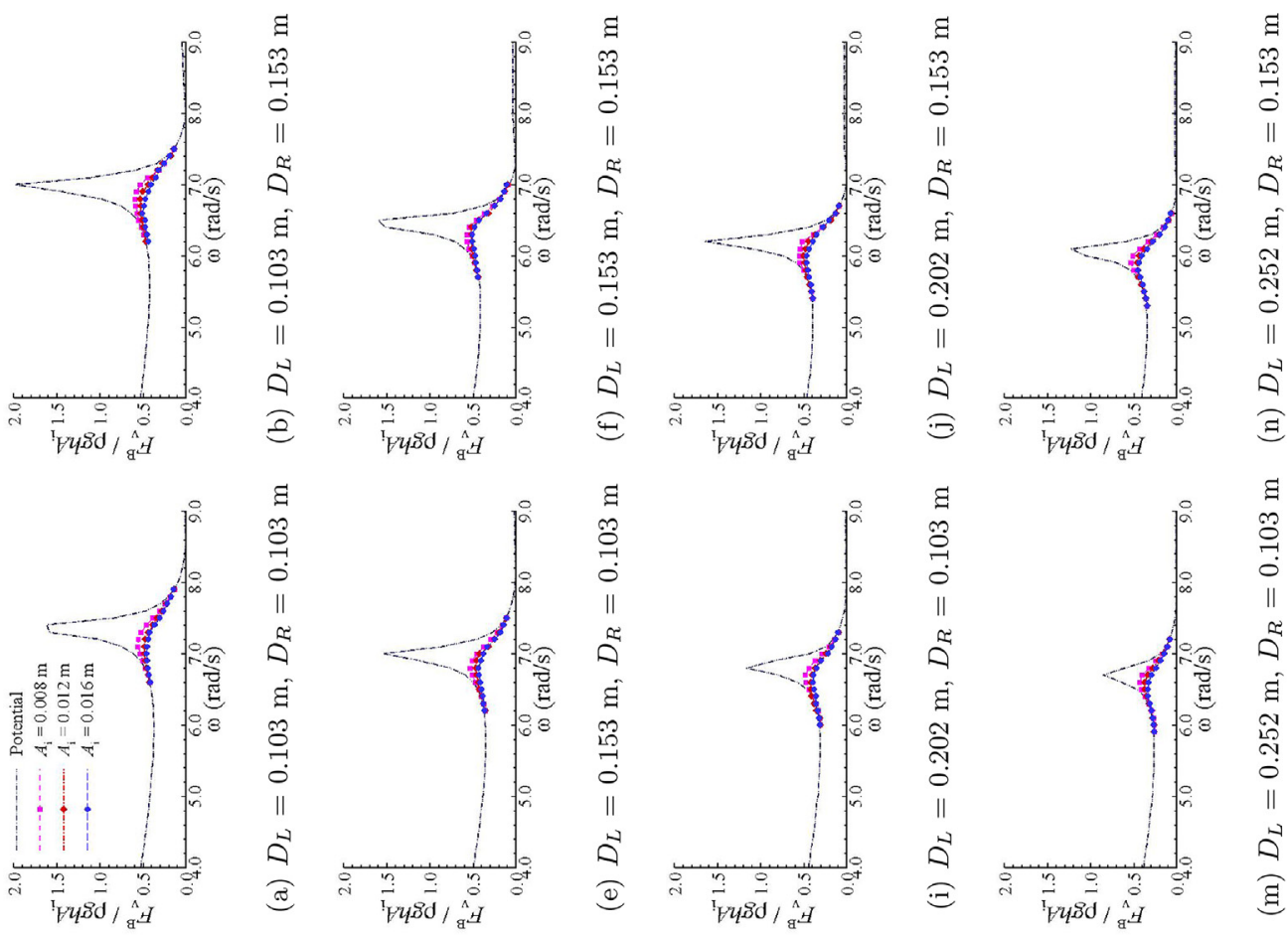

Fig. 25. Comparison of normalized vertical wave forces $F_{v}^{B}$ on Box B under various incident wave amplitudes.

operations of side-by-side FLNG or FPSO with larger draft. In the region considered in the present study a general estimation of 1.1-1.5 times increase by tripling the gap breadth can be suggested for the horizontal wave forces. As for the vertical wave forces on Box B, the magnitudes of resonant forces even approach more than two times when the gap breadth increases from $30 \mathrm{~mm}$ to $90 \mathrm{~mm}$.

With increasing incident wave amplitude, the normalized wave forces on Boxes A and B tend to be smaller around resonant frequencies. It can be speculated that the wave forces are closely relevant to the wave response in the narrow gap. A slight decrease of resonant frequencies of the horizontal and vertical wave forces on Box B can be also observed, which is analogous with that of 
resonant frequencies of wave response in the narrow gap. The large-amplitude piston-type free surface oscillation in the narrow gap is the dominant factor for the wave forces on Box B since the wave motion downstream of Box B is calm. Difference to the results of wave response in the narrow gap, the characteristic/resonant frequencies of wave forces on Box A increase slightly with the increase of incident wave amplitude. The influence of free surface oscillation in the front of Box A is the important reason for this discrepancy. On the whole, the behavior of wave forces has some similarity with that of wave response in the narrow gap, indicating that the largeamplitude free surface oscillation in the gap has significant effect on the wave forces. However, evident discrepancy between them can also be observed because the other factors, such as the wave response upstream and downstream the two-body systems, also have the non-negligible contributions to wave forces.

\section{Acknowledgements}

This work was supported by the Natural Science Foundation of China with Grant No. 51490673, the Fundamental Research Funds for the Central Universities with Grant No. DUT18LK09, and the Pre-research field Fund Project of the Central Military Commission of China with Grant No. 61402070201. The first author gratefully acknowledges the Supercomputer Center of Dalian University of Technology for providing computing resources.

\section{References}

[1] Ananthakrishnan P. Viscosity and nonlinearity effects on the forces and waves generated by a floating twin hull under heave oscillation. Appl Ocean Res 2015;51:138-52.

[2] Berberović E, van Hinsberg NP, Jakirlić S, Roisman IV, Tropea C. Drop impact onto a liquid layer of finite thickness: dynamics of the cavity evolution. Phys Rev 2009;79:036306.

[3] Chen XB. Hydrodynamics in offshore and naval applications-part I. Keynote lecture of 6th intl. Conf. HydroDynamics, Perth (Australia). 2004.

[4] Faltinsen OM, Rognebakke OF, Timokha AN. Two-dimensional resonant piston-like sloshing in a moonpool. J Fluid Mech 2007;575:359-97.

[5] Feng X, Bai W. Wave resonances in a narrow gap between two barges using fully nonlinear numerical simulation. Appl Ocean Res 2015;50:119-29.

[6] Fredriksen AG, Kristiansen T, Faltinsen OM. Experimental and numerical investigation of wave resonance in moonpools at low forward speed. Appl Ocean Res 2014;47:28-46.

[7] Hirt CW, Nichols BD. Volume of fluid (vof) method for the dynamics of free boundaries. J Comput Phys 1981;39:201-25.

[8] Iwata H, Saitoh T, Miao G. Fluid resonance in narrow gaps of very large floating structure composed of rectangular modules. Proceedings of the fourth international conference on asian and pacific coasts, Nanjing, China. 2007. p. 815-26.

[9] Jacobsen NG, Fuhrman DR, Fredsøe J. A wave generation toolbox for the open-source cfd library: openfoam. Int J Numer Meth Fluid 2012;70:1073-88.

[10] Jasak H. Error analysis and estimation for the finite volume method with applications to fluid flows PhD. thesis Imperial College London (University of London); 1996.

[11] Jiang SC, Bai W, Tang GQ. Numerical simulation of wave resonance in the narrow gap between two non-identical boxes. Ocean Eng 2018;156:38-60.

[12] Lu L, Cheng L, Teng B, Zhao M. Numerical investigation of fluid resonance in two narrow gaps of three identical rectangular structures. Appl Ocean Res 2010;32:177-90.

[13] Lu L, Teng B, Cheng L, Sun L, Chen X. Modelling of multi-bodies in close proximity under water waves-fluid resonance in narrow gaps. Sci China Phys Mech Astron 2011;54:16-25.

[14] Lu L, Teng B, Sun L, Chen B. Modelling of multi-bodies in close proximity under water waves-fluid forces on floating bodies. Ocean Eng 2011;38:1403-16.

[15] Molin B. On the piston and sloshing modes in moonpools. J Fluid Mech 2001;430:27-50.

[16] Moradi N, Zhou T, Cheng L. Effect of inlet configuration on wave resonance in the narrow gap of two fixed bodies in close proximity. Ocean Eng 2015;103:88-102.

[17] Moradi N, Zhou T, Cheng L. Two-dimensional numerical study on the effect of water depth on resonance behaviour of the fluid trapped between two side-by-side bodies. Appl Ocean Res 2016;58:218-31.

[18] Newman J. Progress in wave load computations on offshore structures. Invited lecture, 23th OMAE conference, Vancouver, Canada. 2004http://www.wamit. com/publications.

[19] Peric M, Swan C. An experimental study of the wave excitation in the gap between two closely spaced bodies, with implication for lng offloading. Appl Ocean Res 2015;51:320-30.

[20] Rusche H. Computational fluid dynamics of dispersed two-phase flows at high phase fractions PhD. thesis Imperial College London (University of London); 2003.

[21] Saitoh T, Miao G, Ishida H. Theoretical analysis on appearance condition of fluid resonance in a narrow gap between two modules of very large floating structure. Proceedings of the third asia-pacific workshop on marine hydrodynamics, Shanghai, China. 2006. p. 170-5.

[22] Sun L, Eatock Taylor R, Taylor PH. Wave driven free surface motion in the gap between a tanker and an flng barge. Appl Ocean Res 2015;51:331-49.

[23] Zhao W, Wolgamot HA, Taylor PH, Eatock Taylor R. Gap resonance and higher harmonics driven by focused transient wave groups. J Fluid Mech 2017;812:905-39. 